\title{
Contents
}

5 Modelling cystic fibrosis and mucociliary clearance 3

5.1 Mucociliary clearance and cystic fibrosis . . . . . . . . . . . . . . . . . 4

5.1.1 Airway wall environment and mucociliary clearance $\ldots \ldots \ldots \ldots$

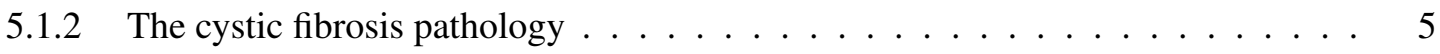

5.1.3 Mathematical modelling of lung wall environment $\ldots \ldots \ldots$

5.2 Newtonian models . . . . . . . . . . . . . . . . . . . . . . . . . . . 10

5.2 .1 Mathematical analysis $\ldots \ldots \ldots \ldots \ldots \ldots$

$5.2 .2 \quad$ Numerical analysis $\ldots \ldots \ldots \ldots \ldots \ldots$

5.2.3 Numerical computations of the mucus propelled by ciliated epithelium . . . . . 21

5.2 .4 Phenomena analysis . . . . . . . . . . . . . . . . . . . 24

5.3 Rheology of mucus and non-Newtonian models $\ldots \ldots \ldots \ldots$

5.3.1 Rheometry data on lung mucus from the literature $\ldots \ldots \ldots . \ldots 26$

5.3.2 Mucus sample analysis and rheological results . . . . . . . . . . . . . . . . . 29

5.3.3 Mathematical modelling of the rheology and related numerical analysis . . . . 33

5.3 .4 Medical outcomes $\ldots \ldots \ldots \ldots \ldots \ldots$

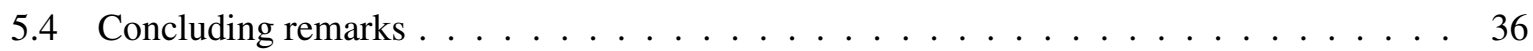




\title{
Chapter 5
}

\section{Modelling cystic fibrosis and mucociliary clearance}

\author{
Dominique Anne-Archard $^{a}$, Robin Chatelin ${ }^{b}$, Marlène Murris-Espin $^{c}$, \\ David Sanchez $^{d}$, Marc Thiriet ${ }^{e}$, Alain Didier ${ }^{c}$, Philippe Poncet ${ }^{f, 1}$
}

${ }^{a}$ Institut de Mécanique des Fluides de Toulouse (IMFT) - Université de Toulouse, CNRS-INPT-UPS, Allée Camille Soula, F-31400 Toulouse, France

${ }^{b}$ Université de Lyon, ENI Saint Etienne, LTDS, UMR 5513 CNRS, 58 rue Jean Parot, F-42023 Saint-Etienne Cedex 2, France

${ }^{c}$ Service de pneumologie et allergologie, CHU de Toulouse, Hôpital Larrey, 24 chemin de Pouvourville, TSA 30030, F-31059 Toulouse Cedex 9, France

${ }^{d}$ Toulouse Institute of Mathematics, UMR CNRS 5219, 135 av. de Rangueil, F-31077 Toulouse, France ${ }^{e}$ EPI INRIA-UPMC-CNRS REO, Université Pierre et Marie Curie, Lab. J-L. Lions, BC 187, 4 place Jussieu, F-75252 Paris, France

${ }^{f}$ University Pau \& Pays Adour, Lab. Mathematics and their Applications (LMAP), UMR CNRS 5142, IPRA, avenue de l'Université, BP 1155, F-64013 Pau, France

${ }^{1}$ Senior author, contact at philippe.poncet@univ-pau.fr 


\subsection{Mucociliary clearance and cystic fibrosis}

This chapter considers the investigation of airway clearance efficiency and dysfunction using rheological measurements, modelling and simulations of mucus flows. The work is mainly dedicated to healthy conditions, respiratory epithelium dysfunction subsequently to the consumption of cilia-inhibiting drugs (eg nicotine) or viral and/or bacterial infections, and cystic fibrosis, although many respiratory diseases are associated with an altered mucus transport.

The first part of this chapter focuses on the trachea-bronchial tree configuration, the respiratory epithelium, the dimensioning aspects and the cystic fibrosis pathology. After a factual dimensioning, the causes and effects of the cystic fibrosis are described. A mathematical modelling is finally built upon the nature of mucus and its surrounding epithelium environment.

The second part is devoted to the Newtonian case: efficient numerical methods are described. The algorithm is based on a penalization technique for the moving cilia-shaped geometry, with a one way coupling (the cell motion is prescribed). The asymptotic analysis and the well-posedness of the mathematical mode are discussed. Specific numerical methods are introduced with their inherent features (convergence, computational time, and memory use) as well as their use when mucociliary clearance is impaired and mucus viscosity rises.

The third part deals with non-Newtonian aspects. Measurements of cystic fibrosis patient mucus is described and summarised, leading to its rheological characterisation. Mixing models for non-Newtonian rheology are based upon a continuous evolution from a low-viscosity Newtonian behaviour, close to the respiratory epithelium wetted surface, to a strongly shear-thinning behaviour in the mucus layer. Finally, an extension of the previous numerical method is set up in order to handle the non-Newtonian features, exhibiting non-reversibility of mucus motion and inefficiency of mucus transport at the cell scale for cystic fibrosis patient.

\subsubsection{Airway wall environment and mucociliary clearance}

The mucus in the human respiratory tract is a non-Newtonian fluid that is secreted by the respiratory epithelium. It protects the respiratory mucosa from dehydration and traps inhaled particles (dust, allergens, carcinogens, micro-organisms, and inflammatory debris). It cleans airways, flowing from either the trachea-bronchial tree and larynx or nose and pharynx to the oesophagus entry, where it is swallowed (or expectorated), as displayed in Figure 5.1. An adequate mucus mobility is necessary for a proper airway clearance. Otherwise pathogens can proliferate and harmful agents can enter the respiratory mucosa.

The airway surface fluid is heterogenous with a near-wall liquid layer containing mostly water and slightly polymerized mucins and a mucus layer in which completely polymerized mucins cross-link and build a meshwork, without discontinuity at the microscopic scale. In addition, the airway surface fluid moves due to the cyclic displacement of cilia. The moving fluid is thus associated with a multi-scale problem both in time and space.

Respiratory ciliated epithelial cells have a collection of cilia on their lumenal (wetted) surface. Over a given small airway segment, these cilia beat uniformly in the periciliary fluid (PCF) at a frequency of 4 to $20 \mathrm{~Hz}$, quite different from that of the respiratory cycle $(0.2-0.25 \mathrm{~Hz})$, during most of their motion cycle. Mucus motion engendered by beating cilia of respiratory epithelial cells is influenced by its geometrical and rheological properties. The viscosity of the mucus film (thickness 10-100 $\mu \mathrm{m}$ ) increases from its outer (close to the respiratory epithelium) to inner part (close to the core air flow).

Later in this section, a description is provided of the model that is used for numerical simulations of the motion of cilia immersed in a heterogeneous fluid with variable viscosity in the direction normal to the wall. The manifestation of cystic fibrosis is characterised by a stagnation of mucus, allowing a 


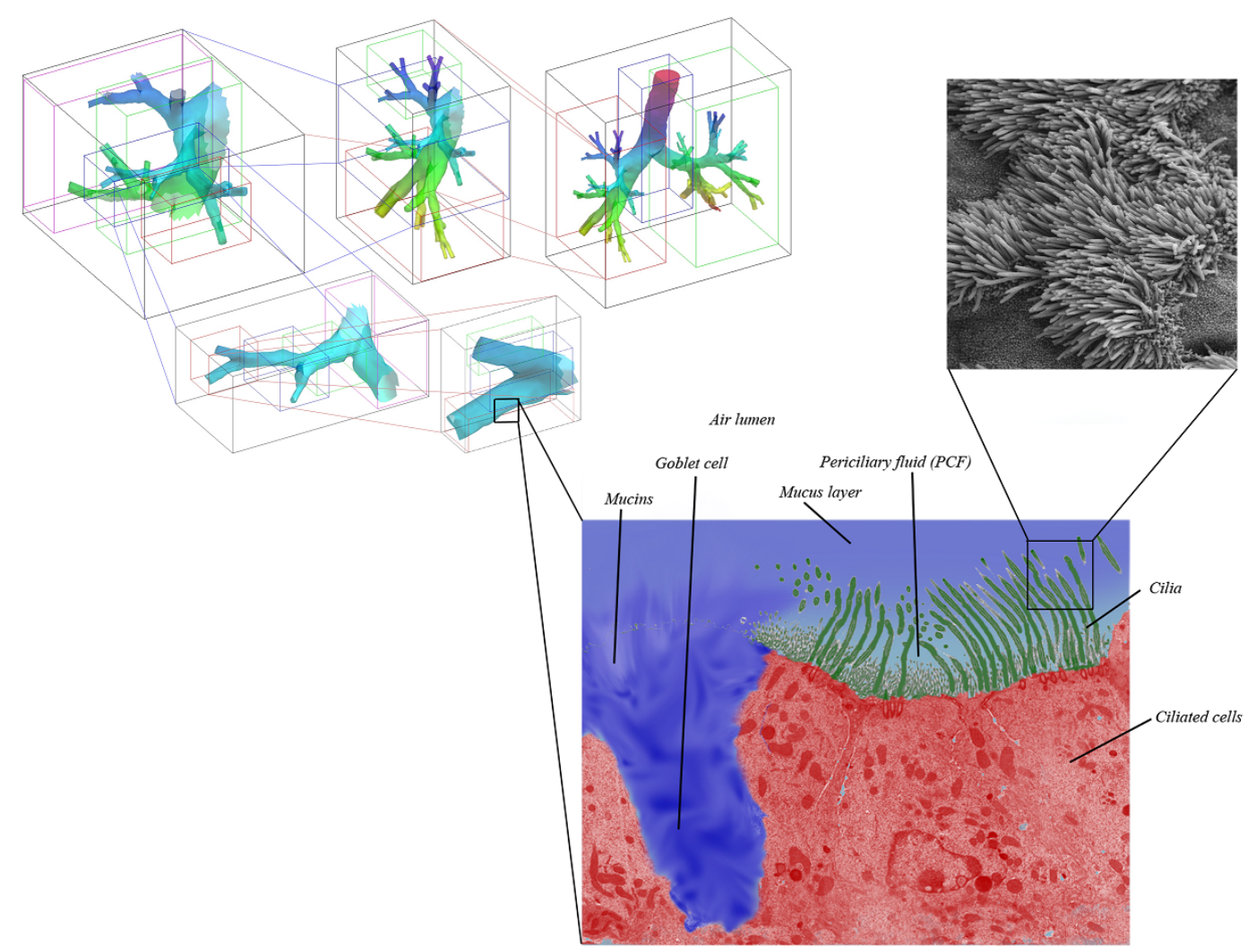

Figure 5.1: Multiscale feature of the tracheobronchial tree along its 6 first generations, from the trachea to the ciliated cells covering a large part of the lung epithelium, taken from Chatelin and Poncet (2013).

proliferation of pathogens agents and thus major infections, whose inflammation effects tend to destroy the epithelium ciliated cells, leading to sharper stagnation. It results that dangerous bacteria, such as Pseudomonas aeruginosa releasing pyocyanin, can then colonise the lung. Computing the resulting fluid displacement enables to study impact of different physical and biological parameters on the mucus transport at the scale of the cell, pointing out mechanisms involved in cystic fibrosis, the major pathological target of the present work.

\subsubsection{The cystic fibrosis pathology}

Cystic fibrosis (CF) is a monogenic autosomal recessive disorder. This most frequent life-shortening genetic disease in the Caucasian population is caused by mutations in the cystic fibrosis transmembrane conductance regulator (CFTR) gene on chromosome 7. These mutations lead to the production of a dysfunctional CFTR protein.

The prevalence of the disease varies according to location and ethnic background. CF affects more than 70,000 people worldwide (30,000 in the USA, 6000 in France). (Hoffman and Ramsey, 2013). It is observed in 1 infant over 2500 in Europe and North America. The prevalence of cystic fibrosis in the United States of America is 0.797 per 10000 inhabitants and in average 0.737 in the 27 European Union countries, with a highest value of 2.98 in the Republic of Ireland (Farrell, 2008).

More than 2000 mutations have been identified in the CFTR gene. Despite the allelic diversity in this genetic disease, about $85 \%$ of White people ${ }^{2}$ with CF pathology carry at least one copy of the CFTR

\footnotetext{
${ }^{2}$ In the general meaning of people from European extraction, including American white people. This aims at being be more general and rigorous than Caucasian, including intermingling since former Europe.
} 
gene mutation $\Delta \mathrm{F} 508$ (F508 ${ }^{\text {del }}$ CFTR channel variant, cf. Quon and Rowe (2016)). Approximately 4\% of all Whites carry a single cystic fibrosis mutation. Carriers of a single cystic fibrosis mutation do not generally exhibit any phenotypic abnormality. Two mutations are required to be present for clinical manifestations of the disease.

In 1969, CF patients in industrialised countries had a mean survival of 14 years. Because of better understanding of relationships between CFTR gene defects and organ pathophysiology, life expectancy has improved substantially over the last 40 years. By 2010 the median predicted survival rate approached 40 years (Clancy and Jain, 2012).

The CFTR protein belongs to the large family of adenine nucleotide-binding cassette transporters consisting of two transmembrane domains, two nucleotide binding domains (NBDs) and a unique regulatory domain. This cAMP-regulated anion channel ${ }^{3}$ is expressed in the airway epithelium, submucosal glands and many other epithelial organs (eg, biliary and pancreatic ducts) (Mall and Hartl, 2014). It transport anions (mainly chloride and bicarbonate) through the apical membrane of epithelial cells, thereby creating an osmotic gradient for fluid secretion; the active ion transport is coupled with water efflux. The basic defect causes an imbalance between secretion and absorption of the epithelial lining fluid rendering CF airway surfaces vulnerable to dehydration. The induced decrease in the thickness of the airway surface liquid is associated with an increased viscosity and thickening of airway secretions, an obstruction of airway lumens, a decreased ability to clear inhaled pathogens, and a dysregulated inflammation.

Studies in cultured primary airway epithelia from CF patients carrying a broad spectrum of CFTR mutations demonstrated that the airway surface liquid is depleted with a collapse of the periciliary layer and a mucociliary dysfunction (Mall and Hartl, 2014). In the following sections, the very different nature between periciliary fluid and mature mucus is investigated, and our results confirm the drop of the mucociliary clearance in the case of PCL collapsing.

Static airway mucus triggers inflammation of epithelial tissue and forms a nidus for bacterial infection. Defective CFTR-mediated bicarbonate transport leads to an acidic airway surface liquid that results in a decrease in antimicrobial activity (Haq et al., 2015). The epithelial sodium channel (ENaC) activity is upregulated in $\mathrm{CF}$, which leads to sodium hyper-absorption and contributes to airway surface dehydration. ENac activation occurs via various mechanisms including the cleavage of its subunit by endogenous proteases. Indeed, a damaging vicious circle of mucus retention, inflammation, infection is created (Hoffman and Ramsey, 2013). Many factors mediate the inflammatory response to chronic bacterial infection such as proinflammatory cytokines (IL-1 $\beta$, IL-6, IL-8, GM-CSF, TNF- $\alpha$ ). Sputum of $\mathrm{CF}$ contains elevated levels of neutrophils and hence exhibits a high concentration of neutrophil-derived proteases such as neutrophils elastase (Twigg et al., 2015).

Clinically, CF is a complex multi-organ disease that leads to significant chronic sinopulmonary disease, pancreatic exocrine and endocrine impairments (diabetes), bowel disease, elevated sweat chloride, and male infertility (Zielenski, 2000). Chronic inflammation and infection may potentiate respiratory tissue injury, thereby altering progressively pulmonary function. The primary cause of death and longterm complications among people with CF is chronic bacterial infection in lungs (Yankaskas et al., 2004). Chronic obstructive lung disease evolves from an early onset of mucus plugging in small airways, chronic neutrophilic airway inflammation and bacterial infection (Zemanick et al., 2010).

Cystic fibrosis patients battle life-long pulmonary infections. Chronic pulmonary infections contributes to a decline in forced expiratory volume in $1 \mathrm{~s}$ (FEV1) of approximately $2 \%$ per year. They result from a limited number of microbial pathogens, among which the most common species are Haemophilus influenza, Staphylococcus aureus, Pseudomonas aeruginosa, and the Burkholderia cepacia complex. CF mucus is a specifically favorable niche for growth of Pseudomonas aeruginosa including glycosylation of airway mucins (Jeffries et al., 2015). There is sufficient evidence that eradication of early infection

\footnotetext{
${ }^{3}$ cAMP stands for cyclic adenosine monophosphate, involved in the cAMP receptor proteins (CRP) binding tightly to a specific DNA.
} 
and prevention of chronic Pseudomonas aeruginosa infection is associated with clinical benefit (Doring et al., 2012).

Until recently, medical treatments for CF exclusively targeted specific sequelae of the underlying pulmonary disease. For example, intravenous or inhaled antibiotics are administered to limit bacterial infection while macrolides or ibuprofen can be used to reduce inflammation (Doring et al., 2012). Restoring the impaired clearance of airway secretions in order to optimize microorganisms removal from airways is one of the major goals of therapy in patients with CF (Kurbatova et al., 2015a).

Current therapies are varied and include: (i) airway clearance to mobilize broncho-pulmonary secretions and interrupt the vicious cycle of inflammation and infection (McShane et al., 2013), (ii) inhaled dornase which while it does break down DNA and decrease mucus viscosity, has recently been shown to requires a peripheral small airway deposit (Kurbatova et al., 2015b), (iii) hypertonic saline which may improve the airway surface hydration (Hoffman and Ramsey, 2013), and (iv) mannitol that can enhance the airway mucociliary clearance (Aitken et al., 2012; Bilton et al., 2011).

New pharmacotherapy approaches focus on the development of CFTR modulators. These CFTRtargeted drugs are designed to rescue mutant CFTR function (Mall and Hartl, 2014). Correctors relieve protein misprocessing due to the most common mutation $\Delta \mathrm{F} 508$, as it facilitates proper maturation and delivery to the plasma membrane. Potentiators are CFTR modulators that increase the time CFTR spends in the open channel configuration and hence chloride transport. They restore airway surface hydration and mucus clearance. Combined therapies (potentiators and correctors) are now available for patients (Quon and Rowe, 2016).

Alternative therapeutic approaches to improve airway surface hydration include stimulation or inhibition of other channels and transporters involved in apical ion transport to obviate the abnormal ion transport, solute transfer, $\mathrm{pH}$ and airway surface liquid volume generated by dysfunctional CFTRs. Given hyperactivity of $\mathrm{ENaC}$ in $\mathrm{CF}$, inhibitors of the ENac channel or activators of alternative $\mathrm{Cl}^{-}$channels $(\mathrm{CaCC})$ have recently been investigated as potential therapies (Haq et al., 2015).

\subsubsection{Mathematical modelling of lung wall environment}

Since the 1970's and the paper of (Blake, 1972), the mucociliary clearance has been frequently studied using mathematical modelling combined with numerical and analytic computations - see (Smith et al., 2008) for a review. Since the publication of this work (2008), several researchers published new researches using 2D modelling (Smith et al., 2007b; Lukens et al., 2010; Montenegro-Johnson et al., 2013; Lee et al., 2011; Jayathilake et al., 2015; Sedaghat et al., 2016) or 3D modelling (Jayathilake et al., 2012; Simons et al., 2015; Smith et al., 2007a; Li et al., 2016).

In these papers the airway surface liquid is often considered to have a two-layer structure with constant viscosity in each layer (Lee et al., 2011; Jayathilake et al., 2015; Li et al., 2016). Alternately, other studies assume a constant viscosity airway surface liquid composed only with one fluid layer (Jayathilake et al., 2012; Simons et al., 2015). The two layer model has also been combined with rheological behaviours in the upper mucus layer, using a shear-thinning (Montenegro-Johnson et al., 2013; Craster and Matar, 2000) or a viscoelastic (Mitran, 2007; Sedaghat et al., 2016) modelling.

Regarding numerical analysis, the mucociliary clearance has been computed using various techniques. As the flow modelling leads to a Stokes problem, the Stokeslets method (Cortez et al., 2005) has been used by several authors (Smith et al., 2007a,b; Lukens et al., 2010; Montenegro-Johnson et al., 2013). The main drawbacks of this method include the computational cost which grows with the number of immersed cilia and becomes prohibitive in $3 \mathrm{D}$, as well as the limitation to constant viscosity flows avoiding the computation of non-linearities such as rheology.

Finite volumes have also been used: a complete discretisation coupled to a finite element beam model 


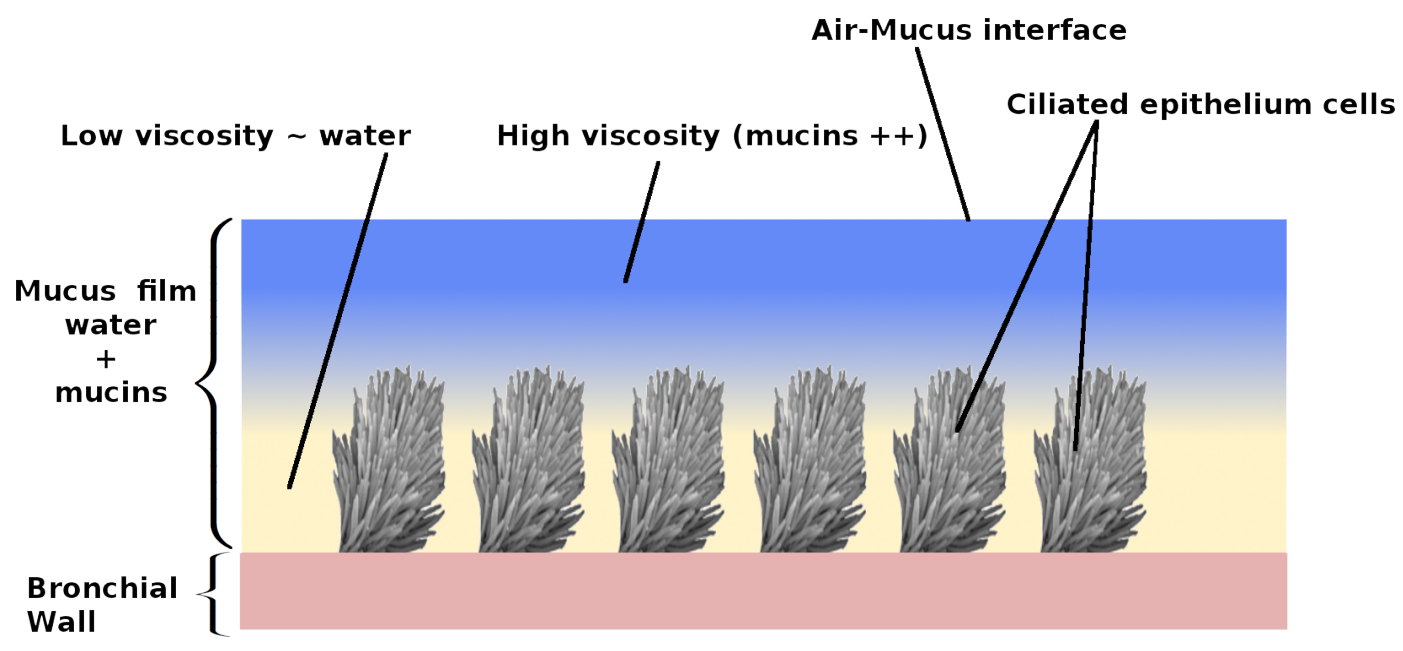

Figure 5.2: Sketch of the mucus flow on the bronchial wall: the non-homogeneous mucus, which exhibits a smooth transition between PCL and maturated mucus, is propelled by beating ciliated epithelium cells, from Chatelin and Poncet (2016).

for cilia has been published (Mitran, 2007), taking into account the fluid-structure interaction. The flow was successfully computed but this technique requires a large amount of computational resources to solve resulting linear systems. The immersed boundary method, introduced by Peskin (Peskin, 2003) was also used in a finite differences context for a parametric study. More recently the immersed boundary method was coupled with Lattice Boltzmann techniques in order to take into account the two-layer structure of the airway surface liquid (Li et al., 2016; Sedaghat et al., 2016).

Because the characteristic dimensions of the mucus flow are very small (the mucus film is $10-40 \mu \mathrm{m}$ height and cilia are $5-8 \mu \mathrm{m}$ long), the inertia terms of the Navier-Stokes equations are negligible and the conservation of momentum can be represented by the linearised Stokes equations. In fact at this scale inertia terms are vanishing in the Navier-Stokes equations. To simulate the mucociliary clearance it is important to take into account the cilia motion which propels the airway surface liquid by mean of fluid-structure interaction. In the following model description, the fluid-structure interaction is assumed to be "one way": the force exerted by the fluid on the solid is not computed. In fact this remains an open problem from a numerical point of view, in particular an eventual beating adaptation (when the airway surface liquid becomes more viscous for example) has never been studied numerically and, to our knowledge, only one experimental work was reported (Hill et al., 2010).

The existence of an interface between the periciliary fluid and mucus is debated (Button et al., 2012; Ehre et al., 2014). In fact, polymerized mucins are released in the periciliary fluid, where they become progressively hydrated during their crossing of the PCL. Mucins accumulate at a given distance normal to the wall, giving rise to a mucin-concentrated phase, the mucus. In this region, mucin polymers interweave and eventually cross-link. The whole set of transformations undergone by mucins during their transport through the ASL is refered to as maturation in the present text.

These mucins are primarily responsible for the mucus structure and its highly non Newtonian behaviour. Near the epithilium the mucin concentration is very low and the airway fluid behaves as a Newtonian fluid, while further away from the epithilium, the presence of high mucin concentrations results in non-Newtanian shear thinning behaviour. In order to represent the airway fluid, and particularity its viscosity, as a continuum instead of a two layer fluid, a variable $\alpha$ is introduced that is a function of position that varies in a smooth sigmoidal manner between 0 and 1 . This function is defined in greater detail later in this section.

This means that the viscosity can be seen as an ad-hoc function of $\alpha$ : Either $\mu=\Phi(\alpha)$ in the 


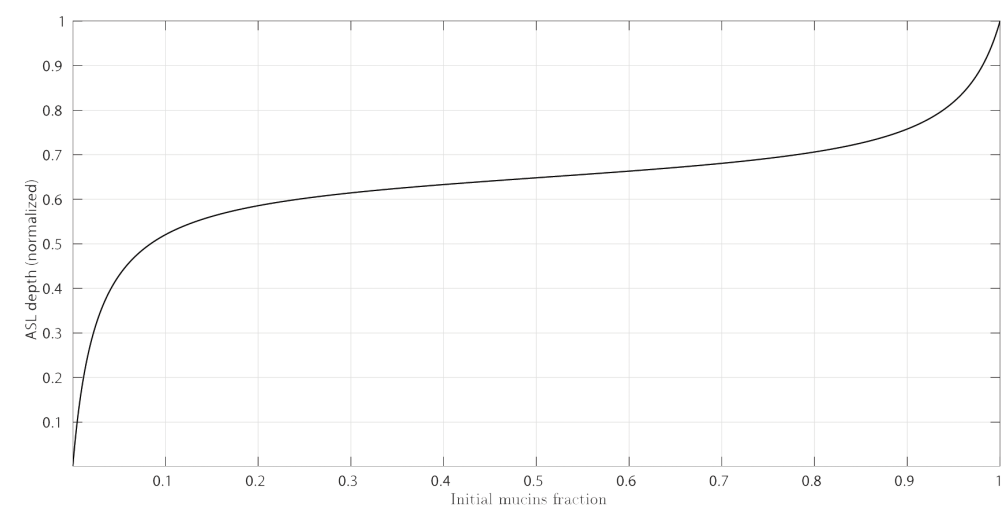

Figure 5.3: Initial profile $\alpha$ used for the cross-linked mucins ratio, defined by Equation (5.3), with respect to the normalised depth $\tilde{z}=z / H, H$ being the total mucus height.

Newtonian case, detailed in Section $\S 5.2$, or $\mu=\Phi(u, \alpha)$ in the non-Newtonian case, typically

$$
\mu=k(\alpha)(2 D: D)^{n(\alpha)-1}
$$

for power-law fluids, or a Carreau law for the fluids whose rheology exhibits thresholds, where $D$ is the strain tensor defined below, detailed in Section \$5.3. Then starting from an initial distribution, this variable $\alpha$ which characterises mucins evolution, is transported and diffused by the mucus flow which depends on the viscosity. Hence $\alpha$ is the solution to a convection-diffusion equation and it is the natural to assume that the viscosity is bounded: $\mu_{0} \leq \Phi(\alpha) \leq \mu_{1}$.

The mathematical problem which has to be solved is the following:

$$
\begin{cases}u=\bar{u} & \text { inside cilia, } \\ -\operatorname{div}(2 \mu D(u))=f-\nabla p & \text { inside fluid, } \\ \operatorname{div} u=0 & \text { inside fluid, } \\ \partial_{t} \alpha+u \cdot \nabla \alpha-\eta \Delta \alpha=0 & \text { everywhere in computational domain } \\ \mu=\Phi(\alpha) \text { or } \mu=\Phi(\alpha, u) & \text { everywhere in computational domain }\end{cases}
$$

where $p$ is the pressure, $\mu$ is the fluid viscosity, $f$ are the external forces and $D(u)=\left(\nabla u+(\nabla u)^{T}\right) / 2$ is the strain tensor and $\bar{u}$ is the cilia velocity (see computational details in \$5.2.2.1). With initial and boundary conditions yet to be set.

To take into account the motion of immersed beating cilia, the Penalisation method is used (Angot et al., 1999). It consists in adding an extra term to the conservation of momentum to solve a unique equation in all the domain, without any distinction between the cilia region $B(t)$ and the fluid region $\Omega \backslash B(t)$ (assuming that $\Omega$ is the computational domain). This extra Penalisation term forces the velocity $u$ to be equal to a prescribed solid velocity $\bar{u}$ in the solid region $B(t)$. It transforms the previous problem into the Penalized Stokes problem or the Stokes-Brinkman problem coupled to the same convectiondiffusion equation generating a non-linear dynamics:

$$
\begin{cases}-\operatorname{div}\left(2 \mu^{\varepsilon} D\left(u^{\varepsilon}\right)\right)+\frac{\mathbf{1}_{B(t)}}{\varepsilon}\left(u^{\varepsilon}-\bar{u}\right)=f-\nabla p^{\varepsilon} & \text { in } \Omega, \\ \operatorname{div} u^{\varepsilon}=0 & \text { in } \Omega, \\ \partial_{t} \alpha^{\varepsilon}+u^{\varepsilon} \cdot \nabla \alpha^{\varepsilon}-\eta \Delta \alpha^{\varepsilon}=0 & \text { in } \Omega, \\ \mu^{\varepsilon}=\Phi\left(\alpha^{\varepsilon}\right) \text { or } \mu^{\varepsilon}=\Phi\left(\alpha^{\varepsilon}, u^{\varepsilon}\right) & \text { in } \Omega,\end{cases}
$$

with $\mathbf{1}_{B(t)}$ the characteristic function of the solid domain and $\varepsilon$ the penalty parameter.

This coupled non-linear problem will be solved using dedicated algorithms in order to get powerful 3D simulations and to allow parametric investigations. As detailed in Section \$5.2.2 these algorithm are 
based on fast solvers on regular Cartesian grids, so that the computational domain $\Omega$ is assumed to be a rectangular cuboid.

The Stokes problem is completed with a no slip boundary condition at the epithelium surface (where the periciliary fluid is present), and a free slip boundary condition at the air-mucus interface (which is assumed to be flat due to a strong surface tension effect (Enault et al., 2010; Chatelin et al., 2016b)) and periodic boundary conditions in the other (proximal and transverse) directions. In the following, let denotes $\Gamma_{0}$ and $\Gamma_{L}$, the upper and respectively lower boundaries of the rectangular cuboid computational domain. Hence $\Gamma_{0}$ and $\Gamma_{L}$ respectively, is the boundary regions where the homogeneous Dirichlet and homogeneous Neumann respectively, boundary conditions are imposed.

For the convection-diffusion equation, homogeneous Neumann boundary conditions are set at both epithelium-periciliary-fluid-layer and air-mucus interfaces (meaning on $\Gamma_{0} \cup \Gamma_{L}$ ) and periodic boundary conditions in other directions.

Moreover an initial conditions is provided for the mucins ratio $\alpha$. A sigmoid profile is used to model the cross-linked mucins ratio which continuously varies from 0 in the deeper periciliary fluid layer to 1 in the upper mucus layer:

$$
\alpha(x, y, z, t=0)=\frac{\arctan (\gamma(z-\delta))-\arctan (-\gamma \delta)}{\arctan \left(\gamma\left(H_{z}-\delta\right)\right)-\arctan (-\gamma \delta)}
$$

where $\delta$ is the ratio between the mucus layer height and the periciliary fluid layer height, and $z$ denotes the coordinate perpendicular to the epithelium designating position in the fluid. A depiction of Equation (5.3) is presented in Figure 5.3.

\subsection{Newtonian models}

A Newtonian fluid behaviour means that the viscosity $\mu$ only depends on $\alpha$, and is independent of $u$ : In the sets of Equations 5.1 and 5.2, the case $\mu=\Phi(\alpha)$ is considered. This section is divided in three part: firstly the well-posedness of Eq. 5.2 is demonstrated, secondly the numerical algorithms dedicated to the computation of this problem are presented, finally several computations are presented as well as phenomenological analysis.

\subsubsection{Mathematical analysis}

In order to perform a mathematical analysis of the problem, we consider first a regularisation of the domain $B(t)$ described in Figure 5.4. From a technical point of view, this allows the use trace and lifting theorems in these two domains and to justify the pertinence of the Penalisation method. For the sake of simplicity the notation $B(t)$ refers to this regularised domain in the whole Section 5.2.1.

Let $T>0$ a final time (not necessarily a cilia period). Let $\Gamma=\left[0, L_{1}\right] \times\left[0, L_{2}\right]$ and $\Omega=\Gamma \times[0, L]$ the domain of study. We denote by $\Gamma_{0}=\Gamma \times\{0\}$ and $\Gamma_{L}=\Gamma \times\{L\}$ the lower and upper boundaries of $\Omega$.

Since the domain $B$ depends on the time parameter $t \in[0, T]$, we need to track this dependency in the following. We let

$O=[0, T] \times \Omega$ the whole domain in time and space coordinates,

$O_{p}=\{(t, x) \in[0, T] \times \Omega, \forall t \in[0, T], x \in B(t)\}$ the penalized domain,

$O_{p}^{c}=\{(t, x) \in[0, T] \times \Omega, \forall t \in[0, T], x \in \Omega \backslash \bar{B}(t)\}=O \backslash \bar{O}_{p}$,

$\Sigma=\{(t, x) \in[0, T] \times \Omega, \forall t \in[0, T], x \in \partial B(t) \backslash \partial \Omega\} \quad$ the inner boundary of $O_{p}$. 


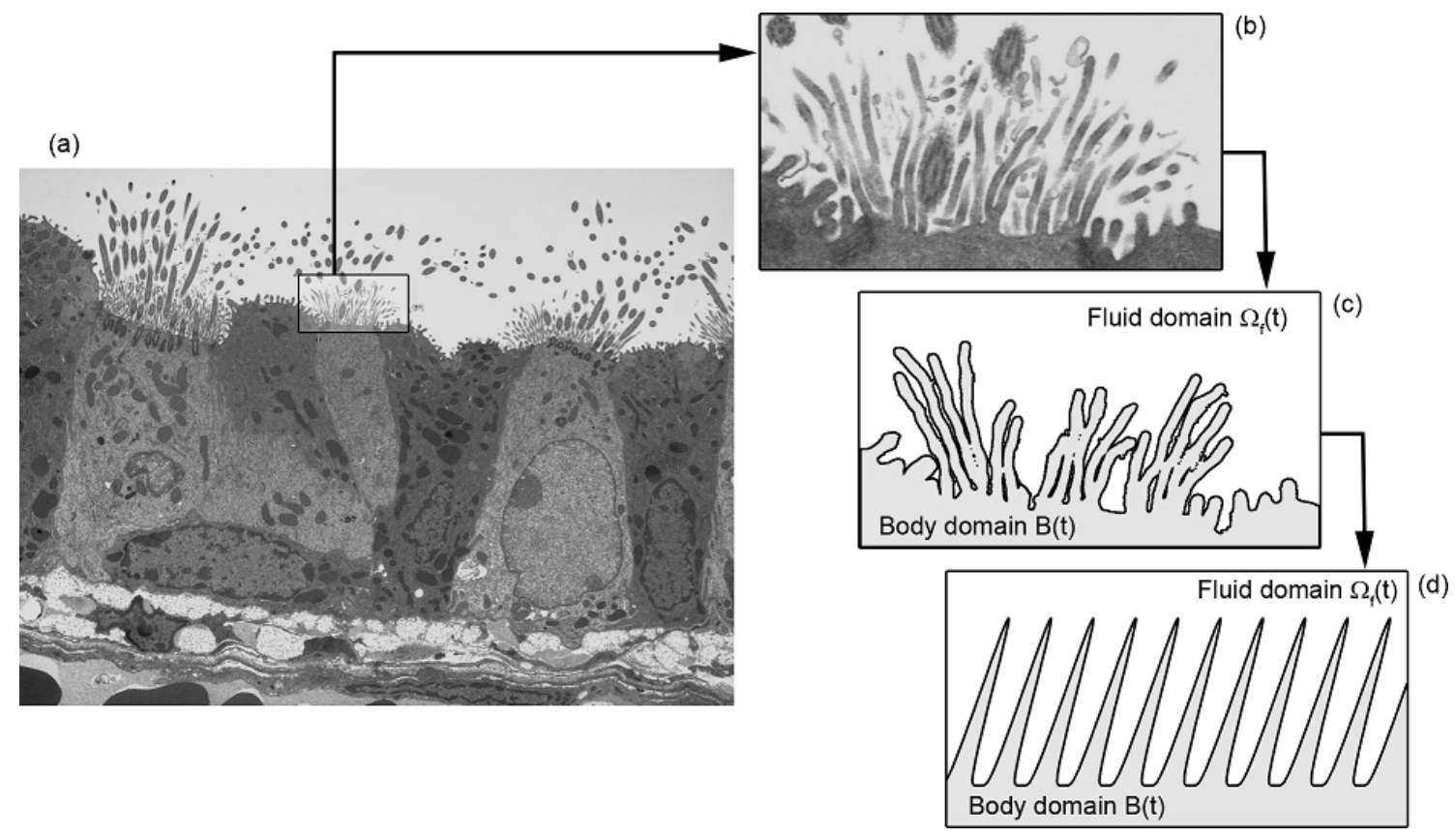

Figure 5.4: The moving domain $B(t)$ models the fixed bronchial wall of the lung and the moving cilia. In the following we consider the model d) where the boundary $\partial B(t)$ is regular (from Chatelin et al. (2016b)).

The problem (5.1) is rewritten in these spaces:

$$
\left\{\begin{array}{l}
u=\bar{u} \text { in } O_{p} \\
-\operatorname{div}(2 \mu D(u))=f-\nabla p \text { in } O_{p}^{c} \\
\operatorname{div} u=0 \text { in } O \\
\partial_{t} \alpha+u \cdot \nabla \alpha-\eta \Delta \alpha=0 \text { in } O \\
\mu=\Phi(\alpha) \text { in } O
\end{array}\right.
$$

with the initial and boundary conditions described previously:

$$
\left\{\begin{array}{l}
u=0 \text { on }[0, T] \times \Gamma_{0} \\
u=\bar{u} \text { on } \Sigma, \\
u \cdot \nu=\frac{\partial u_{1}}{\partial \nu}=\frac{\partial u_{2}}{\partial \nu}=0 \text { on }[0, T] \times \Gamma_{L}, \\
u \text { is periodic on }[0, T] \times \partial \Gamma \times[0, L], \\
\frac{\partial \alpha}{\partial \nu}=0 \text { on }[0, T] \times\left(\Gamma_{0} \cup \Gamma_{L}\right), \\
\alpha \text { is periodic on }[0, T] \times \partial \Gamma \times[0, L], \\
\alpha(0, \cdot)=\alpha_{0} \text { in } \Omega,
\end{array}\right.
$$

where $\eta>0$ is the diffusion coefficient, $\Phi \in \mathrm{W}^{3, \infty}(\mathbb{R})$ and there exists a real number $\mu_{0}>0$ a real such that for all $\alpha \in \mathbb{R} \mu_{0} \leq \phi(\alpha)$.

According to the modelling, the penalized problem is rewritten such that:

$$
\begin{cases}-\operatorname{div}\left(2 \mu^{\varepsilon} D\left(u^{\varepsilon}\right)\right)+\frac{\mathbf{1}_{B(t)}}{\varepsilon}\left(u^{\varepsilon}-\bar{u}\right)=f-\nabla p^{\varepsilon} & \text { in } O \\ \operatorname{div} u^{\varepsilon}=0 & \text { in } O \\ \partial_{t} \alpha^{\varepsilon}+u^{\varepsilon} \cdot \nabla \alpha^{\varepsilon}-\eta \Delta \alpha^{\varepsilon}=0 & \text { in } O \\ \mu^{\varepsilon}=\Phi\left(\alpha^{\varepsilon}\right) & \text { in } O\end{cases}
$$


with its initial and boundary conditions coming straightly from the Equation set (5.5).

$$
\begin{cases}u^{\varepsilon}=0 & \text { on }[0, T] \times \Gamma_{0}, \\ u^{\varepsilon} \cdot \nu=\frac{\partial u_{1}^{\varepsilon}}{\partial \nu}=\frac{\partial u_{2}^{\varepsilon}}{\partial \nu}=0 & \text { on }[0, T] \times \Gamma_{L}, \\ u^{\varepsilon} \text { is periodic } & \text { on }[0, T] \times \partial \Gamma \times[0, L], \\ \frac{\partial \alpha^{\varepsilon}}{\partial \nu}=0 & \text { on }[0, T] \times\left(\Gamma_{0} \cup \Gamma_{L}\right), \\ \alpha^{\varepsilon} \text { is periodic } & \text { on }[0, T] \times \partial \Gamma \times[0, L], \\ \alpha^{\varepsilon}(0, \cdot)=\alpha_{0} & \text { in } \Omega .\end{cases}
$$

Finally the following functional spaces are introduced:

Definition 1 Let $\mathcal{F}(\Omega ; X)$ be the set of functions defined on $\Omega$ with value in $X$ and

$$
\begin{aligned}
& L_{0}^{2}(\Omega)=\left\{v \in L^{2}(\Omega), \int_{\Omega} v d x=0\right\} \\
& V(\Omega)=\left\{v \in H^{1}(\Omega), \operatorname{div} v=0 \text { on } \Omega, u=0 \text { on } \Gamma_{0}\right. \text {, } \\
& \left.u \cdot \nu=0 \text { on } \Gamma_{L}, u \text { has periodic conditions on } \partial \Gamma \times[0, L]\right\}
\end{aligned}
$$

with the classical $H^{1}$-norm. Thanks to the generalized Poincaré's inequality (see (Boyer and Fabrie, 2005) Section III.6), there exist two constants depending only on $\Omega$ such that

$$
c_{1}\|\nabla v\|_{L^{2}(\Omega)} \leq\|v\|_{H^{1}(\Omega)} \leq c_{2}\|\nabla v\|_{L^{2}(\Omega)} .
$$

The following existence and uniqueness results require some regularity assumptions on the obstacle's time evolution (H1)-(H2) and on the velocity $\bar{u}$ inside the obstacle (H3):

\section{Hypothesis 1 (H1)}

- The domain $B$ is an open set in $\Omega$ having the uniform $\mathcal{C}^{2}$-regularity property (see (Adams and Fournier, 2003) Section 4 for the definition) up to the boundary of $\Omega$ in order to obtain a periodic smooth domain,

- $\exists\left(l, l^{\prime}\right) \in\left(\mathbb{R}^{+}\right)^{2}$ such that $0<l<l^{\prime}<L$ and $\Gamma \times[0, l] \subset B \subset \Gamma \times\left[0, l^{\prime}\right]$, i.e. we assume that the bronchial wall is the fixed domain $\Gamma \times[0, l]$ to which cilia are attached and that the cilia never reach the top of the domain $\Omega$,

- $\Omega \backslash \bar{B}$ is an open set in $\Omega$ having the uniform $\mathcal{C}^{2}$-regularity property,

- $\Omega \backslash \bar{B}$ is simply connected.

\section{Hypothesis 2 (H2)}

- There exists a function $\Psi \in \mathcal{C}^{0}\left([0, T] ; \mathcal{C}^{2}(\Omega)\right)$ such that for all $t \in[0, T] \Psi(t)_{\mid[0, l] \times \Gamma}=I d_{[0, L] \times \Gamma}$, $\Psi(t)\left(\left[0, l^{\prime}\right] \times \Gamma\right) \subset\left[0, l^{\prime}\right] \times \Gamma$ and $\Psi(t)$ has periodic boundary conditions on $[0, L] \times \partial \Gamma$,

- $\forall t \in[0, T] \Psi(t)$ is a $\mathcal{C}^{2}$-diffeomorphism on $\Omega$.

- $\forall t \in[0, T] B(t)=\Psi(t, B)$.

Remark: Here the assumption has been made that the bronchial wall never moves and that the cilia never reaches the top of the domain. Additionally: $\Omega \backslash \bar{B}(t)=\Psi(t, \Omega \backslash \bar{B})$. 


\section{Hypothesis 3 (H3)}

- $\bar{u} \in \mathcal{C}^{0}\left([0, T] ; H^{2}(B(\cdot))\right)$

- $\forall t \in[0, T], \operatorname{div} \bar{u}(t, \cdot)=0$ in $B(t)$,

- $\forall t \in[0, T] \bar{u}(t, \cdot)$ fulfills

$$
\forall t \in[0, T] \bar{u}(t, \cdot)_{\mid \Gamma_{0}}=0
$$

- $\forall t \in[0, T] \bar{u}(t, \cdot)$ has periodic boundary conditions on $\partial B(t) \cap(\partial \Gamma \times[0, L])$.

Definition $2\left(u^{\varepsilon}, p^{\varepsilon}, \alpha^{\varepsilon}\right)$ is a weak solution to (5.6)-(5.7) if it fulfills

- $u^{\varepsilon} \in L^{q}(0, T ; V(\Omega)), p^{\varepsilon} \in L^{q}\left(0, T ; L_{0}^{2}(\Omega)\right)$ with $2 \leq q \leq+\infty, \alpha^{\varepsilon} \in L^{\infty}\left(0, T ; H^{2}(\Omega)\right) \cap$ $L^{2}\left(0, T ; H^{3}(\Omega) \cap H^{1}\left(0, T ; H^{1}(\Omega)\right)\right.$,

- for all $v \in \mathcal{C}_{c}(0, T ; V(\Omega))$,

$$
\iint_{(0, T) \times \Omega} 2 \mu^{\varepsilon} D\left(u^{\varepsilon}\right): D(v) d x d t+\frac{1}{\varepsilon} \iint_{O_{p}}\left(u^{\varepsilon}-\bar{u}\right) \cdot v d x d t=\iint_{(0, T) \times \Omega} f \cdot v d x d t
$$

- $\alpha^{\varepsilon}$ is the solution to

$$
\left\{\begin{array}{l}
\partial_{t} \alpha^{\varepsilon}+u^{\varepsilon} \cdot \nabla \alpha^{\varepsilon}-\eta \Delta \alpha^{\varepsilon}=0 \text { in } \Omega \\
\frac{\partial \alpha^{\varepsilon}}{\partial \nu}=0 \text { on } \Gamma_{0} \cup \Gamma_{L}, \\
\alpha^{\varepsilon} \text { has periodic conditions on } \partial \Gamma \times[0, L] \\
\alpha^{\varepsilon}(0, \cdot)=\alpha_{0} \text { in } \Omega
\end{array}\right.
$$

- $\mu^{\varepsilon}=\Phi\left(\alpha^{\varepsilon}\right)$.

Theorem 1 Let $0<\mu_{0}$ and $\varepsilon>0$. Let $\alpha_{0} \in H^{2}(\Omega)$ and $\Phi \in W^{2, \infty}(\mathbb{R})$ such that $\forall x \in \mathbb{R}, \mu_{0} \leq \Phi(x)$. We moreover assume (H1), (H2), (H3) and that for all $T>0, f \in L^{\infty}\left(0, T ; L^{2}(\Omega)\right)$. There exists then a unique weak solution $\left(u^{\varepsilon}, p^{\varepsilon}, \alpha^{\varepsilon}\right)$ to (5.6) such that

$$
\left\{\begin{array}{l}
\alpha^{\varepsilon} \in L^{\infty}\left(0, T ; H^{2}(\Omega)\right) \cap L^{2}\left(0, T ; H^{3}(\Omega)\right) \cap H^{1}\left(0, T ; H^{1}(\Omega)\right), \\
u^{\varepsilon} \in L^{q}(0, T ; V(\Omega)) \text { for all } 2 \leq q \leq+\infty \\
p^{\varepsilon} \in L^{q}\left(0, T ; L_{0}^{2}(\Omega)\right) \text { for all } 2 \leq q \leq+\infty
\end{array}\right.
$$

Moreover there exists some constants independent from $\varepsilon$ such that $u^{\varepsilon}$ fulfills

$$
\begin{aligned}
& \mu_{0}\left\|D\left(u^{\varepsilon}\right)\right\|_{L^{\infty}\left([0, T] ; L^{2}(\Omega)\right)}^{2}+\frac{1}{\varepsilon}\left\|u^{\varepsilon}-\bar{u}\right\|_{L^{\infty}\left([0, T] ; L^{2}(B(\cdot))\right)}^{2} \\
& \leq C\|f\|_{L^{\infty}\left([0, T] ; L^{2}(\Omega)\right)}\|\bar{u}\|_{L^{\infty}\left([0, T] ; H^{2}(B(\cdot))\right)} \\
& +C^{\prime}\left(\|f\|_{L^{\infty}\left([0, T] ; L^{2}(\Omega)\right)}^{2}+\|\Phi\|_{L^{\infty}(\mathbb{R})}^{2}\|\bar{u}\|_{L^{\infty}\left([0, T] ; H^{2}(B(\cdot))\right)}^{2}\right) . \\
& \mu_{0}\left\|D\left(u^{\varepsilon}\right)\right\|_{L^{2}\left([0, T] ; L^{2}(\Omega)\right)}^{2}+\frac{1}{\varepsilon}\left\|u^{\varepsilon}-\bar{u}\right\|_{L^{2}\left([0, T] ; L^{2}(B(\cdot))\right)}^{2} \\
& \leq C\|f\|_{L^{2}\left([0, T] ; L^{2}(\Omega)\right)}\|\bar{u}\|_{L^{\infty}\left([0, T] ; H^{2}(B(\cdot))\right)} \\
& +C^{\prime}\left(\|f\|_{L^{2}\left([0, T] ; L^{2}(\Omega)\right)}^{2}+\|\Phi\|_{\left.L^{\infty}(\mathbb{R})\right)}^{2}\|\bar{u}\|_{L^{\infty}\left([0, T] ; H^{2}(B(\cdot))\right)}^{2}\right) .
\end{aligned}
$$

Definition $3(u, p, \alpha)$ is a weak solution to (5.4) with the conditions (5.5) if it fulfills

- for all $2 \leq q \leq+\infty, u \in L^{q}(0, T ; V(\Omega)), p \in L^{q}\left(0, T ; L_{0}^{2}(\Omega \backslash \bar{B}(\cdot))\right)$ and $\alpha \in L^{\infty}\left(0, T ; H^{2}(\Omega)\right) \cap$ $L^{2}\left(0, T ; H^{3}(\Omega)\right) \cap H^{1}\left(0, T ; H^{1}(\Omega)\right)$, 
- $u=\bar{u}$ in $O_{p}$,

- $(u, p) \in L^{q}\left(0, T ; H^{1}(\Omega \backslash \bar{B}(\cdot))\right) \times L^{q}\left(0, T ; L_{0}^{2}(\Omega \backslash \bar{B}(\cdot))\right)$, with $2 \leq q \leq+\infty$, is the solution to

$$
\left\{\begin{array}{l}
-\operatorname{div}(2 \mu D(u))=f-\nabla p \text { in } L^{2}\left(0, T ; H^{-1}(\Omega \backslash \bar{B}(\cdot))\right), \\
\operatorname{div} u=0 \text { in } O_{p}^{c}, \\
u_{\mid \Sigma}=\bar{u}_{\mid \Sigma} \text { a.e. } \\
u \cdot \nu=\frac{\partial u_{1}}{\partial \nu}=\frac{\partial u_{2}}{\partial \nu}=0 \text { on }[0, T] \times \Gamma_{L}, \\
u \text { has periodic conditions on }[0, T] \times \partial \Gamma \times[0, L],
\end{array}\right.
$$

- $\alpha$ is the solution to (5.8),

- $\mu=\Phi(\alpha)$.

Theorem 2 Under the assumptions of Theorem 1 and with the extra assumption $\Phi \in W^{3, \infty}(\mathbb{R})$, there exists a unique weak solution $(u, p, \alpha)$ to (5.4)-(5.5). Moreover the weak solution $\left(u^{\varepsilon}, p^{\varepsilon}, \alpha^{\varepsilon}\right)$ of (5.6) converges weakly towards $(u, p, \alpha)$ as $\varepsilon$ goes to zero.

We now present the sketches of the proofs of Theorems 1 and 2 and highlight the key points in getting these theorems.

\subsubsection{Sketch of the proof of Theorem 1}

To prove the existence of a solution to this coupled non-linear problem, consider the introduction of the sequence $\left(\alpha^{n}, u^{n}, p^{n}\right)$ defined by:

1. $\alpha^{0}=\alpha_{0}$,

2. $\mu^{n}=\Phi\left(\alpha^{n}\right)$,

3. $\left(u^{n}, p^{n}\right)$ is the solution to:

$$
\left\{\begin{array}{l}
-\operatorname{div}\left(2 \mu^{n} D\left(u^{n}\right)\right)+\frac{\mathbf{1}_{B}(t)}{\varepsilon}\left(u^{n}-\bar{u}\right)=f-\nabla p^{n} \text { in } \Omega, \\
\operatorname{div} u^{n}=0 \text { in } \Omega \\
u^{n}=0 \text { on } \Gamma_{0} \\
u \cdot \nu=\frac{\partial u_{1}}{\partial \nu}=\frac{\partial u_{2}}{\partial \nu}=0 \text { on } \Gamma_{L}, \\
u^{n} \text { has periodic conditions on } \partial \Gamma \times[0, L],
\end{array}\right.
$$

4. $\alpha^{n+1}$ is the solution to:

$$
\left\{\begin{array}{l}
\partial_{t} \alpha^{n+1}+u^{n} \cdot \nabla \alpha^{n+1}-\eta \Delta \alpha^{n+1}=0 \text { in } \Omega \\
\frac{\partial \alpha^{n+1}}{\partial \nu}=0 \text { on } \Gamma_{0} \cup \Gamma_{L} \\
\alpha^{n+1} \text { has periodic conditions on } \partial \Gamma \times[0, L] \\
\alpha^{n+1}(0, \cdot)=\alpha_{0} \text { in } \Omega
\end{array}\right.
$$

We let $\alpha^{n+1}=G\left(\alpha^{n}\right)$.

The study of this recurring sequence leads us to the study of the convection-diffusion equation on one side and the penalized Stokes equation on the other side: 
Theorem 3 Let $\eta>0$ and $u \in L^{q}(0, T ; V(\Omega))$ for all $2 \leq q \leq+\infty$. Let $\alpha_{0} \in H^{1}(\Omega)$. There exists $a$ unique solution $\alpha \in L^{\infty}\left(0, T ; H^{1}(\Omega)\right) \cap L^{2}\left(0, T ; H^{2}(\Omega)\right) \cap H^{1}\left(0, T ; L^{2}(\Omega)\right)$ to the problem (5.8).

Moreover if $\alpha_{0} \in H^{2}(\Omega)$ then $\alpha \in L^{\infty}\left(0, T ; H^{2}(\Omega)\right) \cap L^{2}\left(0, T ; H^{3}(\Omega)\right) \cap H^{1}\left(0, T ; H^{1}(\Omega)\right)$ and there exists some constants $C$ and $C^{\prime}$ depending only on $\Omega$ and $\eta$ such that for all $t \in[0, T]$ :

$$
\begin{aligned}
& \|\alpha(t, \cdot)\|_{H^{2}(\Omega)} \leq C\left\|\alpha_{0}\right\|_{H^{2}(\Omega)} \exp \left(C^{\prime} \int_{0}^{t}\left(1+\|u(s, \cdot)\|_{H^{1}(\Omega)}^{2}+\|u(s, \cdot)\|_{H^{1}(\Omega)}^{4}+\|u(s, \cdot)\|_{H^{1}(\Omega)}^{8}\right) d s\right) \\
& \|\alpha\|_{L^{2}\left(0, t ; H^{3}(\Omega)\right)} \leq C\left\|\alpha_{0}\right\|_{H^{2}(\Omega)} \exp \left(C^{\prime} \int_{0}^{t}\left(1+\|u(s, \cdot)\|_{H^{1}(\Omega)}^{2}+\|u(s, \cdot)\|_{H^{1}(\Omega)}^{4}+\|u(s, \cdot)\|_{H^{1}(\Omega)}^{8}\right) d s\right) .
\end{aligned}
$$

Proof. A classical Galerkin decomposition is performed and energy estimates are made in order to obtain this result (see also Dautray and Lions (2000) Volume 5 Section XVIII).

Theorem 4 It is assumed that hypothesis (H1), (H2) and (H3) are satisfied. Let $\mu_{0}>0, \varepsilon>0$ and $T>0$. Let $\mu \in L^{\infty}([0, T] \times \Omega)$ such that $\mu_{0} \leq \mu(t, x)$ a.e. in $[0, T] \times \Omega$. Let $f \in L^{\infty}\left(0, T ; L^{2}(\Omega)\right)$. There exists then a unique weak solution $(u, p)$ to the problem

$$
\left\{\begin{array}{l}
-\operatorname{div}(2 \mu D(u))+\frac{\boldsymbol{1}_{B(t)}}{\varepsilon}(u-\bar{u})=f-\nabla p \text { in }[0, T] \times \Omega, \\
\operatorname{div} u=0 \text { in }[0, T] \times \Omega, \\
u=0 \text { on }[0, T] \times \Gamma_{0}, \\
u \cdot \nu=\frac{\partial u_{1}}{\partial \nu}=\frac{\partial u_{2}}{\partial \nu}=0 \text { on }[0, T] \times \Gamma_{L}, \\
u \text { has periodic conditions on }[0, T] \times \partial \Gamma \times[0, L]
\end{array}\right.
$$

which fulfills

$$
(u, p) \in L^{q}([0, T], V(\Omega)) \times L^{q}\left([0, T] ; L_{0}^{2}(\Omega)\right)
$$

for all $2 \leq q \leq+\infty$.

Additionally the following inequalities hold:

$$
\begin{aligned}
& \mu_{0}\|D(u)\|_{L^{\infty}\left([0, T] ; L^{2}(\Omega)\right)}^{2}+\frac{1}{\varepsilon}\|u-\bar{u}\|_{L^{\infty}\left([0, T] ; L^{2}(B(\cdot))\right)}^{2} \\
& \leq C\|f\|_{L^{\infty}\left([0, T] ; L^{2}(\Omega)\right)}\|\bar{u}\|_{L^{\infty}\left([0, T] ; H^{2}(B(\cdot))\right)} \\
& +C^{\prime}\left(\|f\|_{L^{\infty}\left([0, T] ; L^{2}(\Omega)\right)}^{2}+\|\mu\|_{L^{\infty}([0, T] \times \Omega)}^{2}\|\bar{u}\|_{L^{\infty}\left([0, T] ; H^{2}(B(\cdot))\right)}^{2}\right) . \\
& \mu_{0}\|D(u)\|_{L^{2}\left([0, T] ; L^{2}(\Omega)\right)}^{2}+\frac{1}{\varepsilon}\|u-\bar{u}\|_{L^{2}\left([0, T] ; L^{2}(B(\cdot))\right)}^{2} \\
& \leq C\|f\|_{L^{2}\left([0, T] ; L^{2}(\Omega)\right)}\|\bar{u}\|_{L^{\infty}\left([0, T] ; H^{2}(B(\cdot))\right)} \\
& +C^{\prime}\left(\|f\|_{L^{2}\left([0, T] ; L^{2}(\Omega)\right)}^{2}+\|\mu\|_{L^{2}\left([0, T] ; L^{\infty}(\Omega)\right)}^{2}\|\bar{u}\|_{L^{\infty}\left([0, T] ; H^{2}(B(\cdot))\right)}^{2}\right) .
\end{aligned}
$$

Moreover, if $\mu \in L^{q}\left(0, T ; W^{1, \infty}(\Omega)\right)$, with $2 \leq q \leq+\infty$,

$$
(u, p) \in L^{q}\left([0, T], H^{2}(\Omega)\right) \times L^{q}\left([0, T] ; H^{1}(\Omega)\right) .
$$

and there exists a constant $C$ depending on $\Omega$ such that

$$
\|u\|_{L^{q}\left([0, T] ; H^{2}(\Omega)\right)}+\left\|\frac{p}{\mu}\right\|_{L^{q}\left([0, T] ; H^{1}(\Omega)\right)} \leq C\|g\|_{L^{q}\left([0, T] ; L^{2}(\Omega)\right)},
$$

where $g=\frac{1}{\mu}\left(2 D(u) \nabla \mu+f-\frac{\mathbf{1}_{B(t)}}{\varepsilon}(u-\bar{u})+p \frac{\nabla \mu}{\mu}\right) \in L^{q}\left([0, T] ; L^{2}(\Omega)\right)$.

Proof. The existence and uniqueness may be obtained classically thanks to Lax-Milgram theorem. The regularity is due to classical Stokes regularity results (see for example Boyer and Fabrie (2005)) thanks to the following rewriting of the equation:

$$
-\operatorname{div} 2 D(u)=\frac{1}{\mu}\left(-2 D(u) \nabla u-\frac{\mathbf{1}_{B(t)}}{\varepsilon}(u-\bar{u})+f-\nabla p\right)
$$


The $\varepsilon$-independent estimates are obtained by taking $u-\tilde{u}$ as test function where $\tilde{u}(t, \cdot)$ is a prolongation of $\bar{u}$ in $\Omega \backslash B(t)$ fulfilling the same boundary conditions as $u$ and the constraint $\operatorname{div} \tilde{u}=0$. To prove the estimates we need time-independent estimates on this prolongation. These ones are obtained thanks to standard time-independent trace and lifting theorem applied to $\bar{u}\left(t, \Psi^{-1}(t, x)\right)$ which is well defined in the non-moving non-deformable domain $B$ thanks to Hypothesis 2. We recover time-independent bounds thanks to the bounds we have on $\Psi$.

Both these results prove that the function $G$ is well-defined from $E \cap F$ in itself with

$$
E=\mathrm{L}^{\infty}\left(0, T ; \mathrm{H}^{1}(\Omega)\right) \cap L^{2}\left(0, T ; \mathrm{H}^{2}(\Omega)\right) \text { and } F=\mathrm{L}^{\infty}\left(0, T ; \mathrm{H}^{2}(\Omega)\right) \cap L^{2}\left(0, T ; \mathrm{H}^{3}(\Omega)\right) .
$$

Moreover when $\alpha$ and $\alpha^{\prime}$ are solutions of (5.8) with the respective velocity $u$ and $u^{\prime}, u$ and $u^{\prime}$ are solutions of (5.9) respectively with the viscosity $\mu$ and $\mu^{\prime}$ and $\mu=\Phi(\alpha), \mu^{\prime}=\Phi\left(\alpha^{\prime}\right)$, we obtain

$$
\begin{aligned}
\left\|\left(\alpha-\alpha^{\prime}\right)(t, \cdot)\right\|_{\mathrm{H}^{1}(\Omega)}^{2} \leq & C\left\|u-u^{\prime}\right\|_{\mathrm{L}^{2}\left(0, t ; H^{1}(\Omega)\right)}^{2} \\
\left\|\left(u-u^{\prime}\right)(t, \cdot)\right\|_{\mathrm{H}^{1}(\Omega)} \leq & C\left\|u^{\prime}(t, \cdot)\right\|_{\mathrm{H}^{2}(\Omega)}\left\|\left(\mu-\mu^{\prime}\right)(t, \cdot)\right\|_{\mathrm{H}^{1}(\Omega)} \\
\left\|\left(\mu-\mu^{\prime}\right)(t, \cdot)\right\|_{\mathrm{H}^{1}(\Omega)} \leq & C\left(1+\left\|\alpha^{\prime}\right\|_{\mathrm{L}^{\infty}\left(0, T ; \mathrm{H}^{2}(\Omega)\right)}\right) \\
& \times\left\|\left(\alpha-\alpha^{\prime}\right)(t, \cdot)\right\|_{\mathrm{H}^{1}(\Omega)} .
\end{aligned}
$$

This leads to

$$
\begin{aligned}
& \left\|\left(G(\alpha)-G\left(\alpha^{\prime}\right)\right)(t, \cdot)\right\|_{\mathrm{H}^{1}(\Omega)} \\
& \leq C\left\|u-u^{\prime}\right\|_{\mathrm{L}^{2}\left(0, t ; H^{1}(\Omega)\right)} \\
& \leq C\left(\int_{0}^{t}\left\|u^{\prime}(t, \cdot)\right\|_{\mathrm{H}^{2}(\Omega)}^{2}\left\|\left(\mu-\mu^{\prime}\right)(s, \cdot)\right\|_{\mathrm{H}^{1}(\Omega)}^{2} d s\right)^{1 / 2} \\
& \leq C\left\|u^{\prime}\right\|_{\mathrm{L}^{2}\left(0, t ; \mathrm{H}^{2}(\Omega)\right)}\left\|\mu-\mu^{\prime}\right\|_{\mathrm{L}^{\infty}\left(0, t ; \mathrm{H}^{1}(\Omega)\right)} \\
& \leq C\left\|u^{\prime}\right\|_{\mathrm{L}^{2}\left(0, t ; \mathrm{H}^{2}(\Omega)\right)}\left\|\alpha-\alpha^{\prime}\right\|_{\mathrm{L}^{\infty}\left(0, t ; \mathrm{H}^{1}(\Omega)\right)} .
\end{aligned}
$$

where the constant $C$ only depends on $T, \Omega, f, \bar{u}, \alpha_{0}, \varepsilon$ and $\|\Phi\|_{\mathrm{L}^{\infty}(\mathbb{R})}$.

Since

$$
\left\|\nabla \mu^{\prime}(t, \cdot)\right\|_{\mathrm{L}^{\infty}(\Omega)} \leq C\left\|\Phi^{\prime}\right\|_{\mathrm{L}^{\infty}(\mathbb{R})}\|\alpha(t, \cdot)\|_{\mathrm{H}^{2}(\Omega)}^{1 / 4}\|\alpha(t, \cdot)\|_{\mathrm{H}^{3}(\Omega)}^{3 / 4},
$$

we obtain that $\mu^{\prime} \in \mathrm{L}^{8 / 3}\left(0, T ; \mathrm{W}^{1, \infty}(\Omega)\right)$ and then that $u^{\prime} \in \mathrm{L}^{8 / 3}\left(0, T ; \mathrm{H}^{2}(\Omega)\right)$. Finally

$$
\left\|u^{\prime}\right\|_{\mathrm{L}^{2}\left(0, t ; \mathrm{H}^{2}(\Omega)\right)} \leq t^{1 / 8}\left\|u^{\prime}\right\|_{\mathrm{L}^{8 / 3}\left(0, T ; \mathrm{H}^{2}(\Omega)\right)} .
$$

There exists then a time $\left.T^{*} \in\right] 0, T[$ such that

$$
\left\|\left(G(\alpha)-G\left(\alpha^{\prime}\right)\right)(t, \cdot)\right\|_{\mathrm{L}^{\infty}\left(0, T^{*} ; \mathrm{H}^{1}(\Omega)\right)} \leq \frac{1}{2}\left\|\alpha-\alpha^{\prime}\right\|_{\mathrm{L}^{\infty}\left(0, T^{*} ; \mathrm{H}^{1}(\Omega)\right)} .
$$

Moreover the time $T^{*}$ only depends on $T, \Omega, f, \bar{u}, \alpha_{0}, \varepsilon$ et $\|\Phi\|_{L^{\infty}(\mathbb{R})}$. Thanks to the estimates in Th. 3 and to the estimates in Th. 4 that are independent from $\alpha$ we obtain the existence of a bound on $\alpha$ valid for all $t \in[0, T]$ and depending only on the data of the problem. Replacing the bounds on $\alpha$ by this new one, we obtain a smaller time $T^{*}$ valid starting from all $t \in[0, T]$.

Since the function $G$ is a contraction on $\left[0, T^{*}\right]$ we obtain that the recurring sequence $\left(\alpha^{n}, u^{n}, p^{n}\right)$ converges to a weak solution on $\left[0, T^{*}\right]$, then on $\left[T^{*}, 2 T^{*}\right]$ and finally on $[0, T]$. Moreover since $G$ is a contraction and thanks to the estimates we have, we prove the uniqueness of the solution.

\subsubsection{Sketch of the proof of Theorem 2}

The solutions of (5.4)-(5.5) are obtained as limit as $\varepsilon$ tends to zero of the solution of (5.6)-(5.7). According to Th. 1 we have that

- $\alpha^{\varepsilon}$ is bounded independently from $\varepsilon$ in the spaces $\mathrm{L}^{\infty}\left(0, T ; \mathrm{H}^{2}(\Omega)\right), \mathrm{L}^{2}\left(0, T ; \mathrm{H}^{3}(\Omega)\right)$ and $\mathrm{H}^{1}\left(0, T ; \mathrm{H}^{1}(\Omega)\right)$, which also implies that $\alpha^{\varepsilon}$ and $\nabla \alpha^{\varepsilon}$ are bounded in $\mathrm{H}^{1}((0, T) \times \Omega)$, 
- $u^{\varepsilon}$ is bounded in $\mathrm{L}^{q}(0, T ; V(\Omega))$ for all $2 \leq q \leq+\infty$,

- $\varepsilon^{-1}\left\|u^{\varepsilon}-\bar{u}\right\|_{L^{q}(0, T ; B(\cdot))}^{2}$ is bounded for all $2 \leq q \leq+\infty$.

The $\varepsilon$-independent bounds makes it possible to extract subsequences still denoted $\left(u^{\varepsilon}, p^{\varepsilon}, \alpha^{\varepsilon}\right)$ that converge weakly or weakly- $\star$ depending on the bounds and such that $u^{\varepsilon}$ converges strongly to $\bar{u}$ in $\mathrm{L}^{q}\left(0, T ; \mathrm{L}^{2}(B(\cdot))\right)$ for all $2 \leq q \leq+\infty$.

One of the key to pass on the limit in nonlinear terms of the equations consists in getting some strong convergence. This one is obtained thanks to the bound on $\alpha^{\varepsilon}$ and $\nabla \alpha^{\varepsilon}$ in $\mathrm{H}^{1}(O)$ which gives thanks to Sobolev injection theorem in $\mathbb{R}^{4}$ the strong convergence of a subsequence of $\alpha^{\varepsilon}$ in $\mathrm{W}^{1, q}(O)$ with $2 \leq q \leq 4$. We now take can take the limit for $u$ in $B(t)$ and in (2) in the domain $\Omega \backslash \bar{B}(t)$ as well as for $\alpha^{\varepsilon}$ in (5.8). Finally the uniqueness of the solution can be gained by adapting the estimates that were used to prove that the function $\mathrm{G}$ defined in the Section §5.2.1.1 is a contraction.

\subsubsection{Numerical analysis}

This section present the numerical algorithms specially developed to compute this mucus flow propelled by the ciliated epithelium cells. The equation set is rewritten:

$$
\begin{cases}\partial_{t} \alpha+u \cdot \nabla \alpha-\eta \Delta \alpha=0 & \text { in } \Omega, \\ \mu=\Phi(\alpha) & \text { in } \Omega, \\ -\operatorname{div}(2 \mu D(u))+\frac{\mathbf{1}_{B(t)}}{\varepsilon}(u-\bar{u})=f-\nabla p & \text { in } \Omega, \\ \operatorname{div} u=0 & \text { in } \Omega,\end{cases}
$$

where all the quantities have the same signification as in the previous section. The $\varepsilon$ indices are removed in this part to improve the algorithms readability.

The first part of this section brings details on the computation of the cilia motion. The second part is dedicated to the computation of the mucins' ratio $\alpha$ (first Equation of (5.10)). The last part details the computation of the fluid velocity, meaning the Stokes problem (both last Equations of (5.10)).

\subsubsection{Computation of the cilia beating}

The cilia motion is decomposed into two phases: the recovery stroke and the effective stroke. The recovery stroke, which lasts about $2 / 3$ of the beating period, cilia are bending and moving backward, close to the bronchial wall and deep in the periciliary fluid layer. During the effective stroke the cilia are more straight and beat forward and penetrating in the mucus layer.

In order to numerically represent a coherent beating, each cilium is modelled by a filament of $0.3 \mu \mathrm{m}$ diameter. The motion of this filament is governed by a transport equation set on a parametric curve which describe the filament centre-line. Equations (5.11) are computed to obtain this, which is presented on the Figure 5.2.2.1, compared with state of the art experimental and numerical works.

Eventually, the beating remains planar in this study and a small decay can be added between two adjacent cilia to create a methachronal synchronisation:

$$
\frac{\partial L}{\partial t}+v(t) \frac{\partial L}{\partial \theta}=0 \quad ; \quad P(0, t)=0 \quad ; \quad L(0, t)=\partial_{\theta} P(0, t)=g(t)=(2 \cos (2 \pi t / T), 0,1)
$$

where $\theta$ is the curve parameter, $P(\theta, t)$ is the curve position at time $t, L=\partial_{\theta} P$ and $v(t)=(1+$ $\left.8 \cos ^{2}(\pi(t+0.25 T) / T)\right) / T$, with $T$ the beating period. To obtain a metachronal synchronisation the same shift is added to both $g$ and $v$. 
(a)

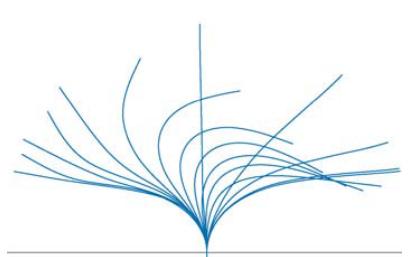

(d) (b)

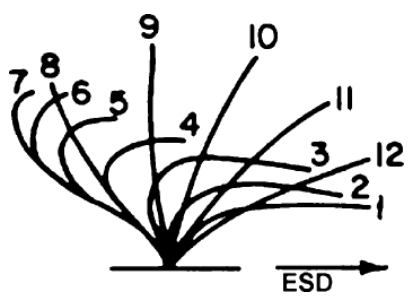

(c)

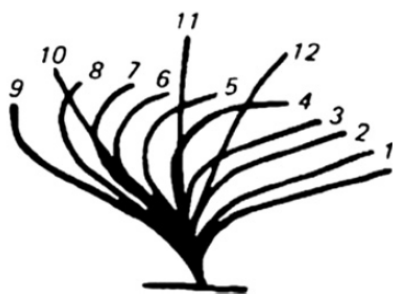

(e)
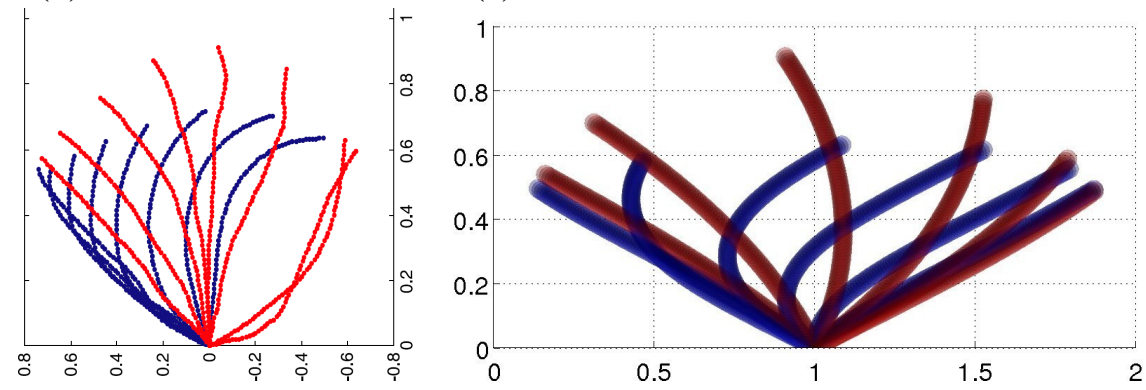

Figure 5.5: Trajectory followed by cilia motion for which each panel is representative of a model posed in the literature: (a) Fauci and Dillon (2006), (b) Gheber and Priel (1997), (c) Sanderson and Sleigh (1981) (culture of rabbit cells), and (d) Mitran (2007) (3D elastic beams model) also representative of the model used in this chapter. Different states of the recovery stroke are displayed in blue, and the states of the effective stroke are displayed in red.

Equations (5.11) enable the computation of the filament position at any time $t$. Then the characteristic function of $B(t)$ the volume occupied by the cilia array can be deduced from the parametric curves, as well as the cilia velocity field $\bar{u}(t)$. These quantities will be used in the following sections.

In all the computations, the counter force applied by the fluid on the solid is not taken into account in the beating model. To our knowledge, this kind of computation and corresponding beating adaptation was only studied experimentally (Hill et al., 2010) and coherent modelling remains an open problem.

\subsubsection{Numerical computation of the mucins convection-diffusion}

The first two Equations of (5.10) can be rewritten as an autonomous dynamical system. Indeed the velocity can be represented a function of the mucins' ratio through the viscosity, $u=F(\alpha)$ :

$$
\begin{cases}\partial_{t} \alpha+F(\alpha) \cdot \nabla \alpha-\eta \Delta \alpha=0 & \text { in } \Omega \\ \mu=\Phi(\alpha) & \text { in } \Omega .\end{cases}
$$

The computation of this non-linear convection-diffusion equation can be difficult in a straightforward manner (finite difference discretisation for example) since two CFL stability conditions arise: one for each phenomenon (convection and transport). Hence the time step must usually be very small imposing a large number of iterations and a large number of linear system assembling and resolution, which is computationally inefficient in 3D.

The viscous splitting (see Beale and Majda (1981)) of Equation (5.12) enables the separation of the resolution of both operators $\partial_{t}+F(\alpha) \cdot \nabla$ and $\partial_{t}-\eta \Delta$, and allows the independent solution of the two problems representing each phenomenon: a linear heat equation for diffusion (which is quite common to solve, particularly when $\eta$ is small enough to permit explicit computations, which is the case in mucus framework) and a non-linear convection equation. In the following simulations the splitting introduced in (Chatelin et al., 2015) guarantees a second order accuracy and permits to alternate the resolution 
of diffusion and convection in order to benefit of diffusion regularising effects after each sub-step of convection.

For this second one a Lagrangian method is used (Cottet and Koumoutsakos, 2000), the mucin's ratio is discretised on a particles cloud:

$$
\alpha(t)=\sum_{p=1}^{\mathcal{P}} \alpha_{p}(t) \delta_{\xi_{p}(t)} v_{p}(t)
$$

where $\xi_{p}$ and $v_{p}$ are respectively the position and the volume of particle $p$ which carries a mucins' ratio $\alpha_{p}$. Using such a discretisation transforms the convection PDE into a set of ODE's:

$$
\frac{d \xi_{p}}{d t}=u\left(\xi_{p}(t), t\right) \quad \frac{d \alpha_{p}}{d t}=0 \quad \frac{d v_{p}}{d t}=0
$$

in the last equation, particles' volume do not change since the velocity field is incompressible.

The advantage solving (5.14) instead of a convection PDE is that there is no CFL restriction, so that large time steps can be achieved. Moreover in (5.14), only the first equation needs to be computed, which is very computationally efficient and is well suited for $3 \mathrm{D}$ computations.

An important feature of the present Lagrangian method is that the velocity field is computed on a Cartesian grid, where the viscosity (and thus mucins' ratio) needs to be known. Hence it is necessary to interpolate these quantities between the grid and the particles. To do that a fourth order interpolation kernel $M_{4}^{\prime}$ (Monaghan, 1985) is used. It guarantees fast computations since the computational cost is also linearly proportional to the number of particles (which is identical to the number of grid points) and accurate results due to the kernel's third order and regularity.

The operator splitting combined with the Lagrangian computation of convection and accurate interpolations with well chosen kernels enables linear computations with respect to the number of grid points. In order to keep the feature, the computations of velocity also needs to be efficient.

\subsubsection{An iterative projection for the incompressible Stokes problem}

The computation of the Stokes problem at any given time $t$ is considered now. The cilia position and velocity are already computed (so $\bar{u}(t)$ and $\mathbf{1}_{B(t)}$ are known) and the convection-diffusion of $\alpha$ was achieved so $\mu(t)$ is also computed. The last two equations of the set in (5.10) remain to be solved:

$$
\begin{cases}-\operatorname{div}(2 \mu D(u))+\frac{\mathbf{1}_{B(t)}}{\varepsilon}(u-\bar{u})=f-\nabla p & \text { in } \Omega, \\ \operatorname{div} u=0 & \text { in } \Omega \backslash B(t),\end{cases}
$$

To compute this problem an iterative projection method has been developed (Chatelin and Poncet, 2013). This class of methods has been widely used for unstationnary Navier-Stokes equations (Guermond et al., 2006). The principle of all these methods is quite similar: the first step consists in solving the momentum equation without the pressure gradient. Hence an intermediate velocity field $u^{*}$ is introduced that is not required to satisfy the incompressibility condition, $\operatorname{div} u=0$.

A projection is then computed and the intermediate velocity is corrected in order to obtain a divergencefree velocity. This is performed by means of the projector $\mathbb{P}\left(u^{*}\right)=u^{*}-\nabla \zeta$ where $\zeta$ is solution of $-\Delta \zeta=-\operatorname{div} u^{*}$ in the whole domain, and satisfying the boundary condition $\partial \zeta / \partial n=u \cdot n$.

The main drawback of these methods is that the projector is usually computed by solving a Poisson problem with an homogeneous Neumann boundary condition. After the correction, the velocity field usually does not satisfy the original boundary condition (Guermond et al., 2006). 
That the boundary conditions are not satisfied becomes more pronounced at low Reynolds numbers. This is the reason that projection methods developed for Navier-Stokes flows cannot be applied to the Stokes equations (Chatelin, 2013). To overcome this problem a new iterative projection method was introduced in (Chatelin and Poncet, 2013). The algorithm is presented as:

1. Initialisation: $u_{0}^{*}=u_{0}$ is provided.

2. Fixed point iterations, where $i$ denotes the iteration number:

(a) $u_{i}^{*}$ is known, as well as $u_{i}=\mathbb{P}\left(u_{i}^{*}\right)$ and $\nabla \zeta_{i}=u_{i}^{*}-\mathbb{P}\left(u_{i}^{*}\right)$,

(b) Prediction : $u_{i+1}^{*}$ is the solution to:

$$
\left\{\begin{array}{lr}
-\mu \Delta u_{i+1}^{*}+\frac{\mathbf{1}_{B(t)}}{\varepsilon}\left(u_{i+1}^{*}-\nabla \zeta_{i}-\bar{u}(t)\right)=f+\left(2 D\left(u_{i}\right)+\operatorname{div} u_{i}^{*}\right) \nabla \mu & \text { in } \Omega \\
u_{i+1}^{*}=g-\nabla \zeta_{i} & \text { on } \partial \Omega
\end{array}\right.
$$

where the last term of (5.16) comes from the substitution $u^{*}=u+\nabla \zeta$.

(c) Correction: compute $\zeta_{i+1}$, the solution to a Poisson problem:

$$
\left\{\begin{array}{lr}
-\Delta \zeta_{i+1}=-\operatorname{div} u_{i+1}^{*} & \text { in } \Omega \\
\frac{\partial \zeta_{i+1}}{\partial n}=0 & \text { on } \partial \Omega
\end{array}\right.
$$

and project: $u_{i+1}=\mathbb{P}\left(u_{i+1}^{*}\right)=u_{i+1}^{*}-\nabla \zeta_{i+1}$.

(d) Repeat $2 \mathrm{a}-2 \mathrm{~b}-2 \mathrm{c}$ and increment $i$ until convergence is satisfied.

3. The solution to the Penalised Stokes problem is $u_{i+1}$.

This algorithm only involves the computation of elliptic problems. In particular the computation of the projector can be achieved using a FFT or multigrid solver (Swarztrauber and Sweet, 1975; Adams, 1989) which are very powerful. Indeed using a regular Cartesian grid the structure of the discretised operator is known a priori and no matrix assembling nor linear system resolution is required. Hence the computational cost is quasi linear with respect to $N$, the number of computational grid points, that is to say with a complexity $\mathcal{O}(N \log N)$. Furthermore it guarantees an accurate computation of boundary conditions, immersed solid velocity, invicid velocity and non-homogeneous effects.

In order to conserve this computational efficiency, which is very attractive for 3D computations, the penalised Stokes Equation (5.16) and the projection using FFT need to be computed with a similar computational cost. The next section describes a numerical method for the penelised Stokes equation using only FFTs.

\subsubsection{A Sherman-Morrison-Woodbury approach to compute Penalised Poisson problems}

To compute the Penalised Poisson problem of at the first step of the previous iterative projection, a special algorithm has been developed (Chatelin and Poncet, 2014), based on the algebraic perturbation relation: the Sherman-Morrison-Woodbury formula (Hager, 1989). This formula enables the computation of the inverse of an invertible perturbed matrix:

$$
\left(A+E^{T} C E\right)^{-1}=A^{-1}-A^{-1} E^{T}\left(C^{-1}+E A^{-1} E^{T}\right)^{-1} E A^{-1}
$$

The problem to solve (first step of the previous iterative projection) can be rewritten as follow (gathering all the term depending on previous iteration $k$ in the RHS):

$$
\left\{\begin{array}{lr}
-\Delta u+c u=f & \text { in } \Omega \\
u=0 & \text { on } \partial \Omega
\end{array}\right.
$$




\begin{tabular}{|c|c|}
\hline Name & Value \\
\hline Cilia length & $8 \mu \mathrm{m}$ \\
Cilia diameter & $0.3 \mu \mathrm{m}$ \\
periciliary fluid viscosity $\left(\eta_{P C F}\right)$ & 0.001 Pa.s \\
Viscosity ratio $\left(\beta=\eta_{M L} / \eta_{P C F}\right)$ & 100 \\
Viscosity transition stiffness $(\gamma)$ & 10 \\
Transition length $(\delta)$ & $7 \mu \mathrm{m}$ \\
airway surface liquid height $\left(H_{z}\right)$ & $13 \mu \mathrm{m}$ \\
Beating frequency & $10 \mathrm{~Hz}$ \\
Mucins diffusion $(\nu)$ & $10^{-11} \mathrm{~m}^{2} . \mathrm{s}^{-1}$ \\
\hline
\end{tabular}

Table 5.1: Reference parameters for the numerical simulations (gathered from (Fahy and Dickey, 2010; Sanderson and Sleigh, 1981; Smith et al., 2008; Thiriet, 2012))

where $c$ represents the Penalisation term and iteration subscripts were removed. Hence this problem can be seen as a Poisson problem perturbed in the cilia volume. Then, applying a generic discretisation to (5.19), it can be exactly rewritten as an algebraic perturbation problem and the SMW formula can be applied:

$$
\left(A+E^{T} C E\right) U=F \Longleftrightarrow U=A^{-1} F-A^{-1} E^{T}\left(C^{-1}+E A^{-1} E^{T}\right)^{-1} E A^{-1} F
$$

In this equation, each product involving $A^{-1}$ can be achieved without matrix assembling and inversion by using the Poisson solver. The key point is to invert the term inside parenthesis without matrix inversion and assembling, which is achieved with an iterative GMRES method. The global algorithm reads in four steps:

1. Solve the Poisson problem $A W=F$

2. Solve $Q Z=E W$ with $Q=C^{-1}+E A^{-1} E^{T}$. This is performed by means of the GMRES method, that requires the product evaluation $Z \rightarrow Q Z$ as follows:

(a) Solve the Poisson problem $A \Theta=E^{T} Z$

(b) Set $Q Z=C^{-1} Z+E \Theta$

3. Solve a last Poisson problem $A Y=E^{T} Z$

4. Update the solution $U=W-Y$

Steps $2 \mathrm{a}$ and $2 \mathrm{~b}$ are computed at each Krylov iteration so the total number of Poisson problems to solve is $2+N_{K r y}$ where $N_{K r y}$ is the typical size of the Krylov space. The other steps are negligible from a computational point of view: as $C$ is a diagonal matrix its inverse is immediately computed, restriction and inclusion matrices $E$ and $E^{T}$ are deduced from $\mathbf{1}_{B(t)}$ and never assembled.

\subsubsection{Numerical computations of the mucus propelled by ciliated epithelium}

The overall algorithm, presented in the previous sections have the following convergence features :

- A $\mathcal{O}(\sqrt{\varepsilon})$ convergence order for the Penalisation method, which is a standard result (Carbou and Fabrie, 2003).

- A second order temporal accuracy, obtained with the splitting algorithm (Chatelin et al., 2015).

- A second order spatial accuracy which is given by the fast FFT solver (Swarztrauber and Sweet, 1975). 


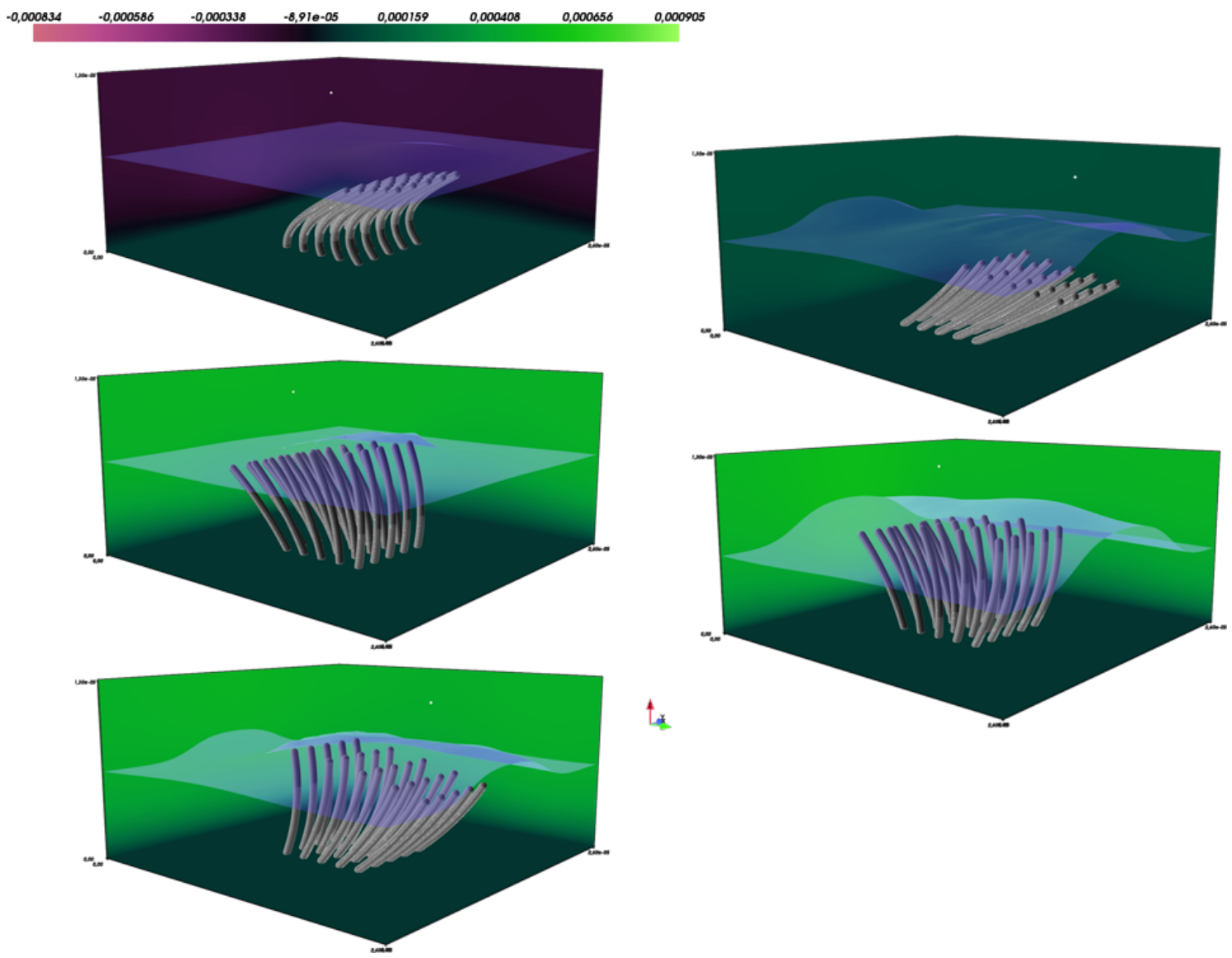

Figure 5.6: Snapshots of the mucus propulsion by a carpet of 25 ciliated epithelium cells at five different instants. The colormap shows the magnitude of the first component of the velocity field. At the beginning of the computation the cross-linked mucins ratio $\alpha$ is initialised according to Equation (5.3). The periciliary fluid viscosity is equal to that of water $\left(10^{-3} \mathrm{~Pa} . \mathrm{s}\right)$ and the viscosity of the mucus is $0.1 \mathrm{~Pa} \cdot \mathrm{s}$. An isosurface of $\alpha=0.5$ is presented in green. In black, a passive particle clearly shows that the mucus is transported toward trachea. Panels (a) to (e) are snapshots at times $t=3.33 \times 10^{-2} \mathrm{~s}, t=0.77 \mathrm{~s}$, $t=0.28 s, t=0.29 s$ and $t=0.88 s$.

- A very negligible mass diffusion in particle transport and interpolation processes (Chatelin and Poncet, 2013)

The first simulation presented involves a simplified epithelium cell composed of 25 cilia regularly arranged as a $5 \times 5$ array. Snapshots are presented in Figure 5.6. This simulation was performed with the parameters gathered of Table 5.1. This figure presents an isosurface of cross-linked mucins ratio, velocity magnitude in the proximal direction and a passive black particle, advected by the flow. On average, this passive tracer travels forward (in the proximal direction to the trachea) which shows that the mucociliary clearance is effective.

The second simulation, presented on Figure 5.7 presents the mucociliary transport with a carpet of 300 cilia, still using the parameters of Table 5.1. This computation presents the effect of the metachronal synchronisation (when a shift is added in the beating between adjacent cilia, creating the metachronal wave) for a large number of cilia. This figure shows that the metachronal wave travels backward, generating a positive first velocity component wave in the mucus layer whereas the velocity is zero or lightly negative in the periciliary fluid layer.

As in the previous figure, the passive black particle clearly shows that the upper part of the airway surface liquid travels forward, thus the mucociliary clearance is efficient. This simulation shows that a 


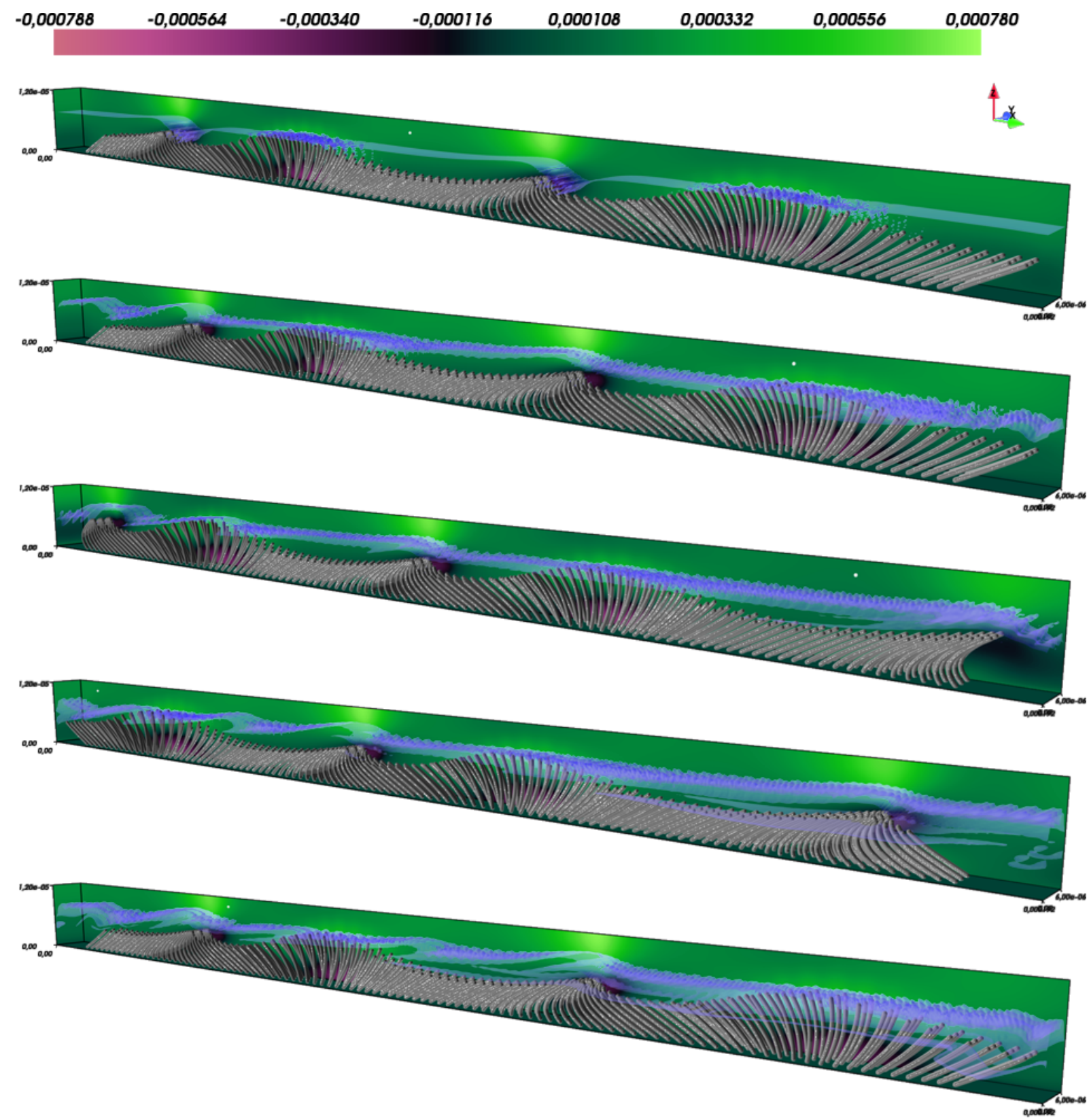

Figure 5.7: Snapshots of the mucus propulsion by a carpet of 300 ciliated epithelium cells at five different times. Colormap and fluid features are the same as in Figure 5.6, except that the isosurface level is $\alpha=0.65$. Panels (a) to (e) are snapshots at times $t=6.67 \times 10^{-3} \mathrm{~s}, t=0.2 \mathrm{~s}, t=0.23 \mathrm{~s}, t=0.35 \mathrm{~s}$ and $t=0.5 \mathrm{~s}$. 
"leading cavity" effect is created when cilia are in recovery stroke, maximising the mucociliary transport. These results, also reported in Hussong et al. (2011) using simplified simulations, help to validate the present methodology.

\subsubsection{Phenomena analysis}

In order to quantify the mucociliary clearance efficiency, the mean velocity of the mucus layer is averaged over several beating periods $T$ :

$$
\bar{U}=\frac{1}{3 T H_{x} H_{y}\left(H_{z}-\delta\right)} \int_{0}^{3 T} \int_{\delta}^{H_{z}} \int_{0}^{H_{y}} \int_{0}^{H_{x}} u_{x}(x, y, z, t) d x d y d z d t
$$

Using different parameter sets, this quantity enables the comparison between different simulations (Chatelin and Poncet, 2016). This can be used in the identification of the meaningful parameters and can help to identify the influence of pathological situations in mucociliary transport. In this study, the following meaningful parameters were identified:

- The beating frequency $2 \pi / T$ is an important parameter of the modelling. Indeed, as a consequence of the time-dilatation of the quasi-static Stokes equations, the mucociliary transport varies linearly with respect to this parameter. This result was also reported using $2 \mathrm{D}$ computations with a bi-fluid modelling (Lee et al., 2011).

- It was also demonstrated that the cilia length is very important. In fact when cilia are too small the mucociliary clearance is deteriorated. This paper shows that it can be divided by ten if cilia are $3 \mu \mathrm{m}$ long instead of 9 . It was demonstrated that the most efficient result is obtained when cilia penetrate the mucus layer during the effective stroke and remain in the periciliary fluid layer during the recovery stroke. This result confirms this assumption formulated in Liron and Rozenson (1983) and contradicts the results of Fulford and Blake (1986).

- As expected, the viscosity ratio between mucus and periciliary fluid has also a great importance: when the mucus becomes highly viscous, the mucociliary clearance is also deteriorated, it can be divided by two when the mucus is 1000 times more viscous than periciliary fluid layer. It is important to notice that the most efficient mucociliary clearance is not obtained for a ratio of 1 (meaning an airway surface liquid only composed of periciliary fluid) but when the mucus is about 10 times more viscous. It means that the mucociliary clearance permits to handle the accumulation of cross-linked mucins in the mucus layer, ensuring a good protection of the airways.

- The transition length $\delta$ between mucus and periciliary fluid layer is also very important. It is the equilibrium between both these characteristic lengths which leads to the penetration (or not) of the cilia tips in the mucus during the effective stroke. In particular when this transition length is too small (this is representative of the case in which nearly all of the airway surface liquid is composed of mucus) the mucociliary clearance can be divided by 2.5 .

- Finally the transition stiffness $\gamma$ between the periciliary fluid layer and the mucus is also an important parameter. It means that to achieve an efficient mucociliary clearance two layers have to be clearly identified. This also enables the penetration in the mucus only during the effective stroke. 


\begin{tabular}{|c|c|c|c|c|c|c|c|c|}
\hline Source & Alias & Rheometer & Pathology & Additional information & $\mathrm{n}$ & $\mathrm{k}$ & $\mathrm{n}^{*}$ & $\mathrm{k}^{*}$ \\
\hline Puchelle et al. (1987) & & $\mathrm{CR}$ & $\begin{array}{l}\text { Chronic airway } \\
\text { hypersecretion }\end{array}$ & & 0.18 & 18.38 & & \\
\hline \multirow[t]{4}{*}{ Jeanneret-Grosjean et al. (1988) } & HL6 & $\mathrm{M} \mu \mathrm{R}$ & Healthy & & - & - & 0.19 & 14.13 \\
\hline & HL7 & & Healthy & Control & - & - & 0.20 & 15.49 \\
\hline & & & Healthy & After administration of Atropine & - & - & 0.20 & 70.79 \\
\hline & & & Healthy & After administration of Diazepam & - & - & 0.15 & 20.42 \\
\hline Rubin et al. (1990) & & $\mathrm{M} \mu \mathrm{R}$ & Healthy & $\begin{array}{l}\text { taken from endotracheal tube } \\
\text { at the end of a surgery }\end{array}$ & - & - & 0.20 & 10.96 \\
\hline \multirow[t]{5}{*}{ Zayas et al. (1990) } & HL8 & $\mathrm{M} \mu \mathrm{R}$ & Healthy & & - & - & 0.22 & 13.18 \\
\hline & & & LS & Without cancer, without obvious airway infection & - & - & 0.17 & 7.08 \\
\hline & & & HS & Without cancer, without obvious airway infection & - & - & 0.15 & 18.62 \\
\hline & & & XS & Without cancer, without obvious airway infection & - & - & 0.16 & 6.92 \\
\hline & & & $\mathrm{C}$ & Without obvious airway infection & - & - & 0.15 & 11.75 \\
\hline Moores et al. (1992) & & CR & COPD & infective exacerbation & 0.04 & 15.00 & 0.13 & 1.71 \\
\hline Dawson et al. (2003) & CFL5 & CR & $\mathrm{CF}$ & - & 0.05 & 18.00 & 0.17 & 7.34 \\
\hline Nielsen et al. (2004) & & $\mathrm{CR}$ & $\mathrm{CF}$ & - & 0.27 & 5.40 & 0.163 & 12.45 \\
\hline \multirow{2}{*}{ Redding (2008) } & & & $\mathrm{CF}$ & Children & - & - & 0.16 & 33.43 \\
\hline & & & $\mathrm{CB}$ & Adults & - & - & 0.14 & 53.03 \\
\hline Innes et al. (2009) & & $\mathrm{CR}$ & Healthy & $\begin{array}{c}\text { Sputum induction performed with } \\
\text { nebulized 3\% saline for } 20 \text { minutes }\end{array}$ & - & - & 0.18 & 3.34 \\
\hline \multirow[t]{3}{*}{ Serisier et al. (2009) } & HL9 & CR & Healthy & Sputum induction with $4.5 \%$ hypertonic saline & - & - & 0.48 & 0.35 \\
\hline & CFL1 & & $\mathrm{CF}$ & Clinically stable & - & - & 0.41 & 0.54 \\
\hline & & & COPD & Clinically stable & - & - & 0.34 & 1.29 \\
\hline Suk et al. (2009) & & CR & $\mathrm{CF}$ & - & 0.16 & 2.42 & & \\
\hline \multirow[t]{3}{*}{ Daviskas et al. (2010) } & & CR & $\mathrm{Br}$ & Clinically stable & - & - & 0.41 & 36.31 \\
\hline & & CR & $\mathrm{Br}$ & Clinically stable & - & - & 0.45 & 24.76 \\
\hline & & & & - After repetitive voluntary coughing - & & & & \\
\hline Schuster et al. (2013) & & $\mathrm{CR}$ & Healthy & $\begin{array}{l}\text { taken from endotracheal tube } \\
\text { at the end of a surgery }\end{array}$ & - & - & 0.16 & 12.97 \\
\hline Tomaiuolo et al. (2014) & CFL2 & CR & $\mathrm{CF}$ & FEV $1 \%>50 \%$ & 0.23 & 2.18 & 0.18 & 1.40 \\
\hline \multirow[t]{2}{*}{ according to FEV1 } & CFL3 & & $\mathrm{CF}$ & $30 \%<$ FEV $1 \%<50 \%$ & 0.21 & 12.81 & 0.17 & 2.25 \\
\hline & CFL4 & & $\mathrm{CF}$ & FEV $1 \%<30 \%$ & 0.26 & 6.87 & 0.16 & 2.42 \\
\hline $\begin{array}{l}\text { Tomaiuolo et al. (2014) } \\
\text { according to main colonization }\end{array}$ & & CR & $\mathrm{CF}$ & S. aureus & 0.20 & 3.04 & - & - \\
\hline
\end{tabular}

Table 5.2: Mucus rheological characterization : power-law parameters. CR: Classical rheometer, M $\mu$ R: Magnetic $\mu$-rheometer. CF: Cystic Fibrosis, Br: Bronchiectasis, COPD: Chronic Obstructive Pulmonary Disease, CB: Chronic Bronchitis, LS: Light Smokers, HS: Heavy Smokers, XS: ExSmokers, C: Cancer. FEV1\%: ratio of the Forced Expiratory Volume in 1 second (FEV1) to the Forced Vital Capacity (FVC). 


\subsection{Rheology of mucus and non-Newtonian models}

\subsubsection{Rheometry data on lung mucus from the literature}

Looking at studies concerned with rheological characterisations of pulmonary mucus, two points are noticeable. First, two kinds of equipment are used: as expected, classical rheometers, but also magnetic micro-rheometers. Secondly rheological characterisations are often limited to the determination of the dynamic storage modulus $\left(G^{\prime}\right)$ and loss modulus $\left(G^{\prime \prime}\right)$ for two frequencies, 1 and $100 \mathrm{rad} / \mathrm{s}$, which are supposed to be characteristic frequencies of mucociliary clearance and cough clearance respectively (App et al., 1998).

\subsubsection{The different measurement methods}

Considering rheological measurements on lung mucus, there are almost as many studies with magnetic micro-rheometers as ones with classical rheometers. The magnetic micro-rheometer uses either a single steel bead (App et al., 1998; Redding, 2008) or multiple magnetite particles (Edwards and Yeates, 1992; Ziemann et al., 1994) positioned in a few $\mu L$ of fluid and oscillated by means of an electromagnetic field to obtain a sinusoidal force. In the first case, the bead diameter ranges from 50 to $100 \mu m$ while particles dimension ranges from 2 to $6 \mu \mathrm{m}$ in the second case. The motion of the single bead or of the particles embedded in the fluid is then quantified, generally by fast image processing techniques: See King and Macklem (1977) and Ziemann et al. (1994) for the detail of rheological parameters extraction. Note that King and Macklem (1977) has incorporated Thomas and Walters (1966) results to take into account elasticity of the fluid. This technique has largely been used for respiratory mucus (Jeanneret-Grosjean et al., 1988; Rubin et al., 1990; App et al., 1998; Zayas et al., 1999; Redding, 2008).

The main advantage of the micro-rheometer lies in the very small volume of fluid that is necessary (few $\mu L$ ). This is of particular interest for mucus that are generally not available in large quantities except for major pathologies such as cystic fibrosis. It is important to note that in general, mucus type fluids are not homogenous anf that larger sample volumes would allow a more representative measurement of the global rheological behaviour and a better precision. Additional drawbacks are that only oscillatory experiments are possible with such a micro-rheometer and that the flow is not a simple shear flow as it is theoretically required for rheometry (Barnes et al., 1989).

Classical rheometry is predominantly used in recent years (Sanders et al., 2000; Dawson et al., 2003; Nielsen et al., 2004; Innes et al., 2009; Serisier et al., 2009; Daviskas et al., 2010; Tomaiuolo et al., 2014). It is performed using most of the time controlled-stress rheometer equipped with cone and plate geometry or parallel plates geometry. These are generally chosen to minimise the fluid volume: small diameter /small angle for the cone $\left(1\right.$ or $2^{\circ}$ ), or small gap for parallel plates (between 50 and $250 \mu m$ when specified).

Note that the term "micro-rheology" may be confusing as it is also used to represent length scales below $0.1-0.3 \mu \mathrm{m}$, for instance to examine virus or nano-particle motion through mucus (Sanders et al., 2000; Dawson et al., 2003; Lai et al., 2009; Schuster et al., 2013). Viscosity experienced by these objects is close to that of water and this is linked to the mesh size of the network structure of mucus which is larger than or similar to the object size. Nevertheless for the mucus clearance process, larger length scales are relevant. So the viscosity that has to be considered here is a macroscopic one which is anticipated to greatly exceed the viscosity of water and is associated to a complex rheological behaviour. 


\subsubsection{Mucus rheology from literature}

A general presentation of mucus, its role, its chemical chemical structure and its physiological properties can be found in Cone (2005). Reviews of their rheological properties are presented by Yeates et al. (1997) and Picout and Ross-Murphy (2003). Considering human airway mucus, there is very little data available in literature for steady state viscosity while data for dynamic viscosity $\eta^{\prime}=G^{\prime} / \omega$ or complex viscosity $\left|\eta^{*}\right|=\left|G^{*}\right| / \omega$, from oscillatory measurements, are more numerous. When it is reported, the measurement temperature is $20^{\circ} \mathrm{C}, 25^{\circ} \mathrm{C}$ or $37^{\circ} \mathrm{C}$. This might appear as a large discrepancy but variances caused by temperature differences are small compared to those from the non-Newtonian feature, as it was demonstrated by Tomaiuolo et al. (2014).

When a rheogram is proposed, all authors agree to recognise a strong shear thinning behaviour. Some authors (Moores et al., 1992; Dawson et al., 2003; Suk et al., 2009) observed a tendency to a Newtonian plateau at low shear rates with a transition towards power-law behaviour between $0.1 \mathrm{~s}^{-1}$ and $1 \mathrm{~s}^{-1}$, depending on authors. On the other hand Yeates et al. (1997) did not observe any trend to a plateau although exploring shear rate from $0.2 s^{-1}$ upwards. The same observation was made by Puchelle et al. $(1985,1987)$ for shear rate starting from $0.01 s^{-1}$ and $0.1 s^{-1}$ respectively. In the shear thinning region, the behaviour can be modelled by a power-law $\tau=k \dot{\gamma}^{n}$, where $\tau$ and $\dot{\gamma}$ are the shear stress and the shear rate respectively, $k$ the fluid consistency and $n$ the power-law index. Values for $k$ and $n$ given in literature, or extracted from literature data, are reported in Table 5.2. Note the low values of the power-law index (between 0.04 and 0.26 ) which are characteristic of a strong shear-thinning behaviour.

Thixotropy is the property of a fluid to show a reversible decreasing viscosity when submitted to a constant shear rate (i.e. observation of a decreasing shear stress as a function of time). Note that thixotropy is sometimes confused with shear-thinning, although rheological definition is unambiguous (Picout and Ross-Murphy, 2003). Thixotropy leads to a hysteresis phenomena that has been observed on viscosities measured at increasing shear rates followed by measurements at decreasing shear rates (Nielsen et al., 2004; Tomaiuolo et al., 2014). Conversely, note that hysteresis is not necessarily evidence of thixotropy because elasticity may also be responsible for hysteresis, especially when the characteristic time of the fluid is comparable to or larger than the time scale of measurement. Mention of the characteristic time for mucus are not numerous in the literature: Besseris and Yeates (2007) gave $42 s$ for canine tracheal mucus, while, for human mucus, Moores et al. (1992) obtained $20 s$ from creep tests and Nielsen et al. (2004) estimated it of the order of thousands of seconds from oscillatory measurements. Academic thixotropy measurements were realized by Puchelle et al. $(1985,1987)$ who observed a shear stress overshoot after a shear rate step. Such an overshoot is linked to elasticity, but successive experiments with decreasing rest time after previous shearing show a decreasing overshoot. This has allowed Puchelle et al. to identify the presence of thixotropy for a reconstituted mucus (Puchelle et al., 1985) and a native mucus (Puchelle et al., 1987).

A few authors have identified a viscoplastic behaviour with a yield stress varying between 0.05 to 0.26Pa (Edwards and Yeates, 1992) until $100 \mathrm{~Pa}$ (Silberberg, 1983). For instance this last value was used by (Craster and Matar, 2000) to analyse numerically the surfactant transport of mucus film in the pulmonary airways. More recently, Broughton-Head et al. (2007) proposed a value of $14 \mathrm{~Pa}$ for cystic fibrosis mucus. As far as we know, these are the only publications identifying a viscoplastic behaviour and giving yield stress values. This can be connected to specific rheometers used or to the way rheological characterisations were conducted.

On the other hand, elasticity has been clearly identified in literature. This is due in part to the widespread use of the magnetic micro-rheometer which is restricted to small-amplitude oscillatory shear experiments. The storage modulus $G^{\prime}(\omega)$ and the loss modulus $G^{\prime \prime}(\omega)$ are the most common data encountered in the literature at the two frequencies $\omega=1 \mathrm{rad} / \mathrm{s}$ and $\omega=100 \mathrm{rad} / \mathrm{s}$. The moduli $G^{\prime}$ and $G^{\prime \prime}$ are the imaginary and real parts of the complex modulus $G^{*}=\tau / \gamma$, where $\gamma$ is the deformation. A phase shift between shear stress and shear rate $\dot{\gamma}$, i.e. a non-zero $G^{\prime}$ value, demonstrate the elastic feature of the fluid. Data are expressed in terms of modulus or, in equivalent form, using the complex modulus 

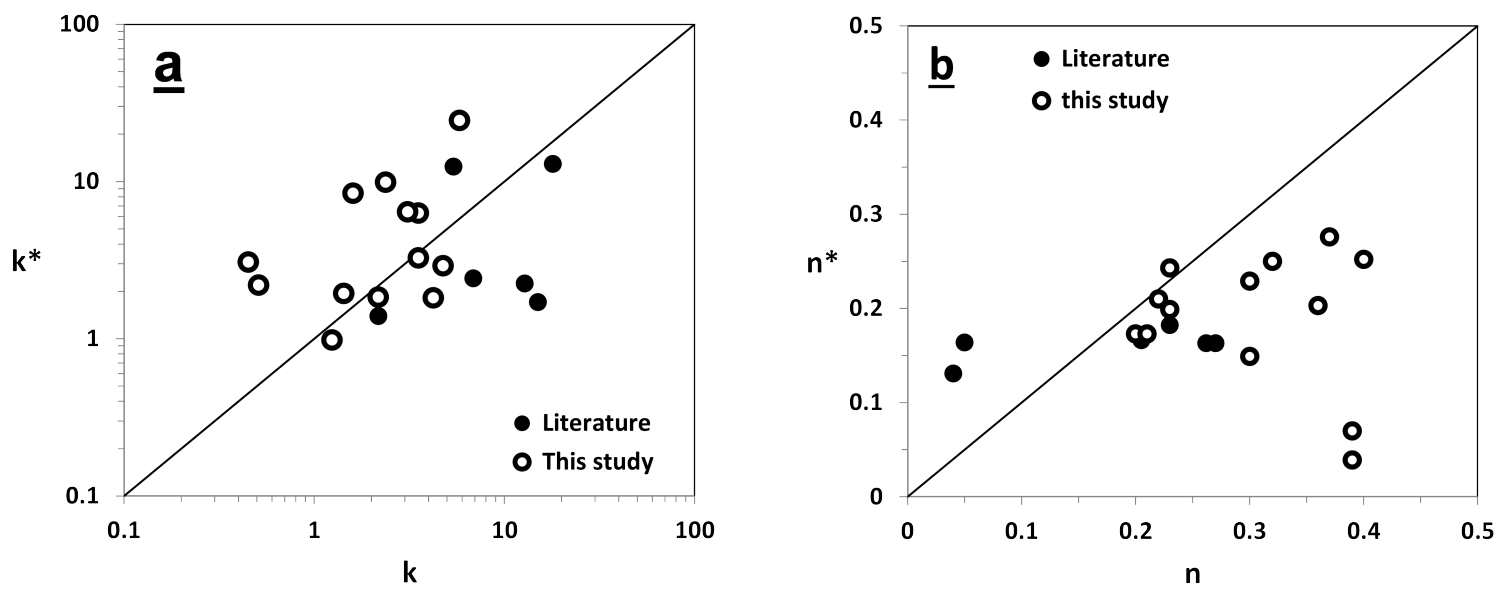

Figure 5.8: Comparison between consistency $k$ and power-law index $n$ issued from viscosity curves and estimations $\left(k^{*}, n^{*}\right)$ issued from the Cox-Merz rule. Note that each point from literature is a mean value while a point marked "this study" corresponds to one of the tested mucus. The solid line denotes the identity.

$G^{*}$ and the loss tangent $\operatorname{tg} \delta=G^{\prime \prime} / G^{\prime}$, or $G^{\prime}$ and the so-called dynamic viscosity $\eta^{\prime}=G^{\prime \prime} / \omega$. The specific two values of the frequency, 1 and $100 \mathrm{rad} / \mathrm{s}$, are often reported in literature because they were proposed by some authors (Jeanneret-Grosjean et al., 1988; Zayas et al., 1990; Shah et al., 1996; App et al., 1998; Zayas et al., 1999; Innes et al., 2009) to define a mucociliary clearability index (conventionally called $M C I$ ) and a cough clearability index $(C C I)$, which quantify respectively the clearability by normalized ciliary function (using values at $1 \mathrm{rad} / \mathrm{s}$ ) and by cough (using values at $100 \mathrm{rad} / \mathrm{s}$ ). As far as we know, the first time a mucociliary clearability index is proposed was by Jeanneret-Grosjean et al. (1988). $M C I$ and $C C I$ are then mentioned in Zayas et al. (1990). Note that these index were also used by Tomaiuolo et al. (2014) at a frequency of $10 \mathrm{rad} / \mathrm{s}$ for $C C I$. These indexes are of particular interest in the evaluation of and quantification of the influence of pathologies on the mucus clearance for smokers and lung cancer patients (Zayas et al., 1990, 1999), for asthma (Innes et al., 2009), and for the influence of treatments or physiotherapy (Shah et al., 1996; App et al., 1998).

In the study of the flow of mucus, the essential parameter is the viscosity. One can ask whether the Cox-Merz rule is sufficient to evaluate $\eta(\dot{\gamma})$ using oscillatory measurement data. The Cox-Merz rule is considered as very useful when only linear viscoelastic data are available (Bird et al., 1977). It is expressed as:

$$
\left|\eta^{*}(\omega)\right|=\sqrt{\eta^{\prime}(\omega)^{2}+\eta^{\prime \prime}(\omega)^{2}}=\left.\eta(\dot{\gamma})\right|_{\dot{\gamma}=\omega}
$$

The Cox-Merz rule is well-adapted for polymer solutions with entangled network structure. It fails (with generally $\left|\eta^{*}\right|>\eta$ ) for cross-linked systems or for highly concentrated entangled systems. Crosslinked polymers are characterised by non-existence of a Newtonian plateau for $\eta(\dot{\gamma})$ and a weak evolution of elastic and viscous modulus with frequency. Based on such observations numerous authors think that mucus is of cross-linked type (Puchelle et al., 1985; Innes et al., 2009; Schuster et al., 2013) but Yeates et al. (1997) developed arguments for an entangled "sliplinked" strand system. Looking at Table 5.2, there are only six cases where the power-law parameters $(n, k)$ and $\left(n^{*}, k^{*}\right)$ (from power-law model applied to $\eta^{*}(\omega)$ available data) can be estimated and compared. The consistency $k$ is underestimated when assimilated to $k^{*}$ for five of the six cases, while no real tendency can be observed on $\left(n, n^{*}\right)$ data (see fig. 5.8). This point will be re-examined later.

Information related to pathology are presented in Figure 5.9. For this representation, the rheological variables $\left|G^{*}\right|$ and $\tan \delta$ were chosen as they are often considered as relatively independent while $G^{\prime}$ and $G^{\prime \prime}$ are not (Jeanneret-Grosjean et al., 1988; King, 1980). Nevertheless, $\left|G^{*}\right|$ varies with respect 


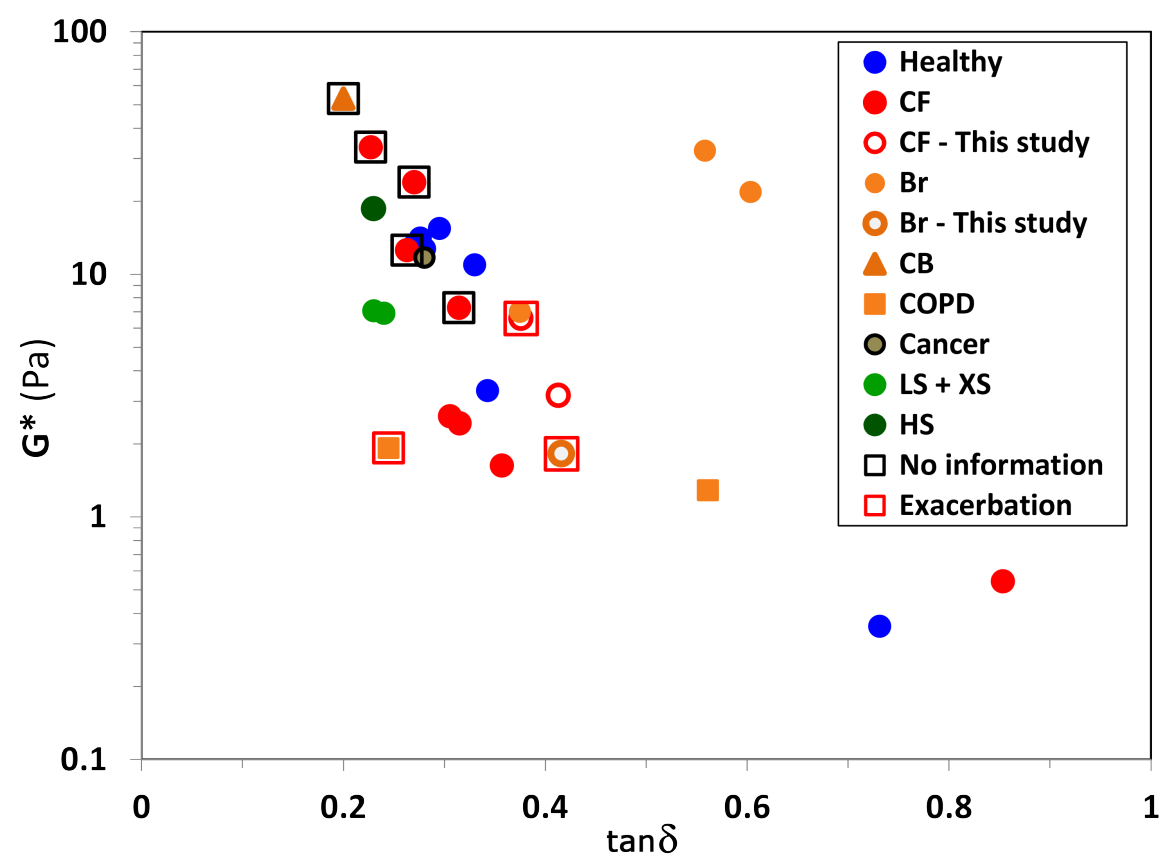

Figure 5.9: Rheological parameters $G^{*}$ and $\tan \delta$ (measured at $1 \mathrm{rad} / \mathrm{s}$ ) for different pathologies. Symbols: same as in Table 5.2. All cases with simple symbols concern studies with patients in a stable state. Symbols with a surrounding square indicate study for patients in exacerbation or study without information.

to viscosity while $\tan \delta$ compares viscous and elastic response of the fluid (with $\tan \delta$ infinite for purely viscous fluids). Concerning healthy patients, it is noticeable that the two points corresponding to the lower $\left|G^{*}\right|$-value were obtained after nebulisation with a saline solution to induce sputum. This probably led to the thinning of the mucus and also helps to explain these lower values as well as the large tan $\delta$ value. Although these comparisons have to be considered cautiously because conditions differ between studies (how mucus is taken and prepared, how rheological characterisations are conducted for example). It is noteworthy to point out that that mucus samples from patients with pathology do not strongly differ with samples from healthy patients (except in some cases of bronchiectasis and COPD). On this graph, the majority of data corresponds to stable patients, or healthy, one case corresponds to exacerbated situation and no information is given for five cases. In a study on rheological properties of CF mucus, (King, 1981) proposed to classify mucus from their visual aspect in three categories (linked to the nature and to the degree of infection): mucoid, muco-purulent and purulent. Comparing $G^{\prime}$ and $\eta^{\prime}$ for three frequencies, he observed that rheological properties for mucoid and muco-purulent human CF mucus are similar to control canine tracheal ones while purulent mucus show higher values and a diminution of $t g \delta$ (i.e. higher elasticity). This was also underlined by Deneuville et al. (1997); Lopez-Vidriero and Reid (1978). Although this distinction between mucus seemed no longer considered for several years, more recent studies have confirmed the interest of this observation, using stable/exacerbated distinction rather than visual criteria : Serisier et al. (2009) and Tomaiuolo et al. (2014) for CF sputum, and Tabatabaei et al. (2015) for COPD.

\subsubsection{Mucus sample analysis and rheological results}

\subsubsection{Mucus samples collecting}

Consecutive adult patients with cystic fibrosis $(\mathrm{CF})$ bronchiectasis $(\mathrm{Br})$ were recruited when admitted in the regional adult $\mathrm{CF}$ center of Toulouse. After information and oral consent, sputum samples were 


\begin{tabular}{c|ccccccc} 
& CFM1 & CFM2 & CFM3a & CFM3b & CFM4 & CFM5 & CFM6 \\
\hline $\mathrm{n}$ & 0.20 & 0.39 & 0.32 & 0.39 & 0.30 & 0.30 & 0.23 \\
$\mathrm{k}$ & 5.80 & 0.45 & 1.24 & 1.60 & 3.53 & 2.37 & 4.22 \\
\hline$T_{c}(s)$ & $>20$ & $\gg 20$ & $\gg 20$ & - & $>20$ & $>20$ & $\approx 20$ \\
\hline $\mathrm{n}^{*}$ & 0.17 & 0.07 & 0.25 & 0.04 & 0.15 & 0.23 & 0.24 \\
$\mathrm{k}^{*}$ & 24.6 & 3.08 & 0.98 & 8.45 & 6.32 & 9.92 & 1.82
\end{tabular}

\begin{tabular}{c|cccccc} 
& CFM7 & CFM8 & CFM9 & CFM10 & CFM11 & BM12 \\
\hline $\mathrm{n}$ & 0.21 & 0.23 & 0.37 & 0.36 & 0.22 & 0.40 \\
$\mathrm{k}$ & 3.10 & 3.53 & 0.51 & 2.17 & 4.75 & 1.43 \\
\hline$T_{c}(s)$ & $\approx 100$ & $\approx 100$ & $\approx 50$ & $\approx 30$ & $>20$ & $\approx 80$ \\
\hline $\mathrm{n}^{*}$ & 0.17 & 0.20 & 0.28 & 0.20 & 0.21 & 0.25 \\
$\mathrm{k}^{*}$ & 6.43 & 3.28 & 2.20 & 1.85 & 2.92 & 1.95
\end{tabular}

Table 5.3: Power-law parameters, relaxation time and power-law parameters from Cox-Merz rule from collected samples. $(n, k)$ data are from Chatelin et al. (2016a).

collected under supervision of a chest physiotherapist. Patients were classified as either having a stable status or having and exacerbation. The classification of exacerbation represented an increased cough or an increased bronchorrhea or purulence sputum, and/or an increased dyspnea, and/or a tiredness and/or a loss of weight). Sputum were frozen at $-20^{\circ} \mathrm{C}$ before rheological analysis.

\subsubsection{Mucus sample analysis}

In this study rheological characterisations were evaluated on a Mars III rheometer (Thermo Sc.) equipped with a cone and plate geometry $\left(1^{\circ}\right.$ angle, $35 \mathrm{~mm}$ diameter) which requires $0.2 \mathrm{~mL}$ of fluid. Measurements were conducted at $37^{\circ} \mathrm{C}$. The lower plate Peltier system for temperature regulation was completed by a sample hood that was custom made in-house with a specific temperature regulation and wet sponges to saturate the measuring chamber and prevent evaporation of the sample. As previously mentioned, samples were stored frozen at $-20^{\circ} \mathrm{C}$. The influence of freezing on the rheological properties of mucus has previously been investigated by Rubin et al. (1990), Sanders et al. (2000), Gastaldi et al. (2000) and Serisier et al. (2009) for different conditions (temperature, freezing duration). These authors all conclude that congelation does not affect rheological behaviour. This result was confirmed in the current study on three samples that were characterized when fresh and again at 21 or 31 days under frozen conditions. Mucus were thawed keeping them in a water-ice mixture just before and during measures to prevent degradation (Sanders et al., 2000).

Once a sample was loaded in the rheometer, the following protocol was followed: an initial dynamical strain sweep at a fixed frequency of $1 \mathrm{~Hz}$ was conducted to determine the linear domain. Then the storage modulus $G^{\prime}(\omega)$ and the loss modulus $G^{\prime \prime}(\omega)$ were determined using an oscillatory frequency sweep for a fixed strain in the linear domain (between $1 \%$ and $3 \%$ according to the sample). After that, the sample was submitted to very large deformationsin which the viscosity $\eta(\dot{\gamma})$ was determined by a steady-shear flow test for shear rates in the range $10^{-3} \mathrm{~s}^{-1}$ to $10^{3} \mathrm{~s}^{-1}$. Careful attention was paid to reaching the steady state regime for cases with low shear rate values. Additional measurements were sometimes conducted to examine the stability of a sample. A power-law model $\eta=k \dot{\gamma}^{n-1}$ ( $k$ : consistency, $n$ : power-law index) was used to fit the rheological data. When the sampled volume was sufficient, two or three different tests were performed. 

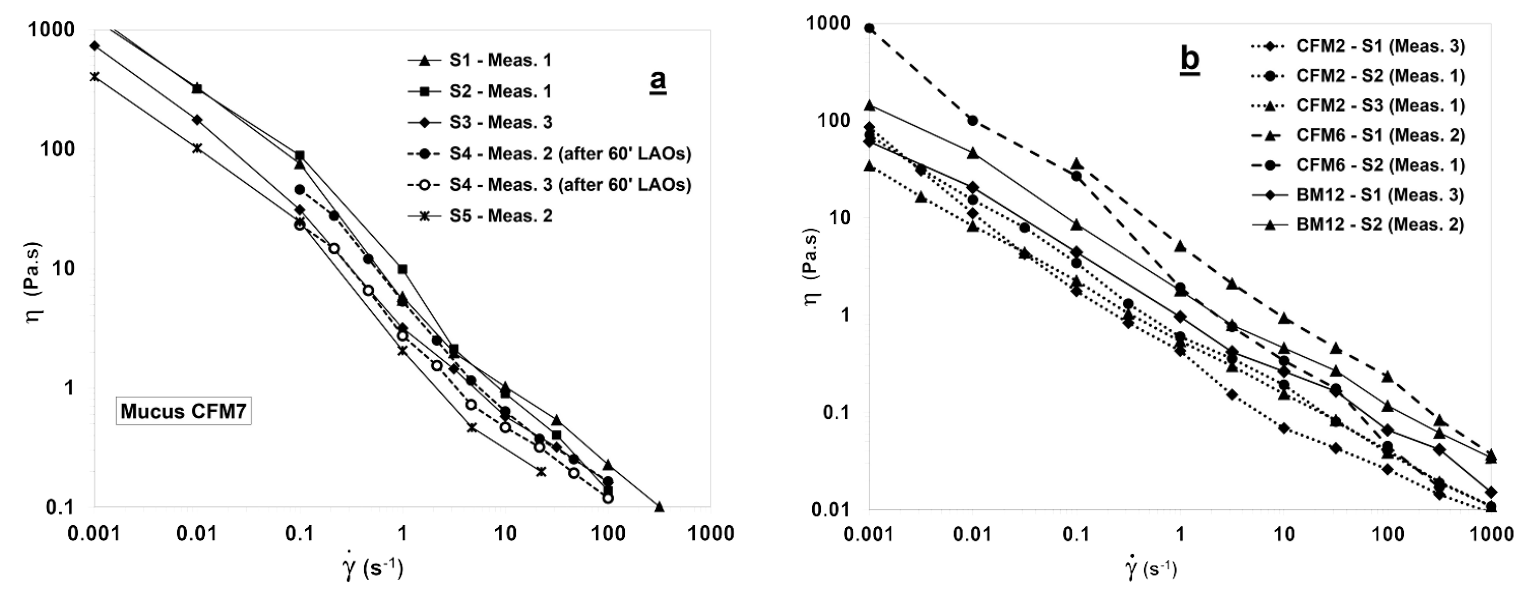

Figure 5.10: Viscosity curves, on the left for mucus CFM7: 'S' standing for Sample and 'Meas.' for measurement. Measurements on sample 4 were done after a sequence of large amplitude oscillations (LAOs) at $300 \%$ and $1 \mathrm{~Hz}$ during 60 minutes. Right picture shows rheograms for three different mucus.

\subsubsection{Rheological results}

Initially a few samples were used in order to determine the measurement strategy. Then fifteen mucus samples were analysed. Two of the samples were rejected because they gave rise to a large dispersion in viscosity and modulus values. Thirteen mucus were then considered for data analysis.

To examine thixotropy, experiments were conducted that were based on the observation of the shear stress overshoot after a shear rate step (similar to those of Puchelle et al. (1987)). Because the results of those experiments were found to be inconclusive, another approach has been adopted. On one of the mucus (CFM7), viscosity measurements were conducted on four samples while on a fifth one, a large amplitude (300\%, i.e. far from the linear domain) oscillatory sequence (LAOs) was first applied during 60 minutes. After that a viscosity measurement was done. The results are reported in Figure 5.10-a. The viscosities for samples S1, S2, S3 and S5 reveals the variability between samples and between measurements. Figure 5.10) reports the data indicating which of the three measurement numbers are reported. For measurements 2 and 3 (Meas. 2 and Meas. 3), only small amplitude oscillatory measurements were conducted before on this sample (strain sweep and/or frequency sweep). No specific classification linked to solicitations to which the sample was previously submitted appears in these results, especially after a strong and long solicitation such as 60 minutes of LAOs (S4, Meas.2 and Meas. 3). Figure 5.10-b presents steady-state shear viscosity curves established for mucus CFM2, CFM6 and BM12. For each of them, two or three measurements were conducted thus illustrating both the dispersion observed on a same mucus and the variability observed between mucus. The dispersion observed on all these series are similar, which implies that thixotropy is weak and comparable here to the natural dispersion arising from heterogeneity of samples and from the measurement difficulties.

As previously reported (Chatelin and Poncet, 2016), a strongly shear-thinning behaviour is observed all over the shear rate range studied. Mucus samples were characterised on a wide shear rate range starting at $10^{-3} \mathrm{~s}^{-1}$ and no trend to a Newtonian plateau was observed, corroborating the results of Puchelle et al. $(1985,1987)$ and Yeates et al. (1997). Note that the same observation can be made on the viscosity calculated from the Cox-Merz rule, i.e. from data for which the fluid has only been subjected to small deformations (in this case, the equivalent shear rate range covered is $\left[0.01 s^{-1} ; 30 s^{-1}\right]$ ). This behaviour is commonly associated with cross-linked polymers and is sometimes referred to as "yield stress" behaviour (leading to the commonly used term "viscoelastic gel"). While data for shear rates as low as $10^{-3} s^{-1}$ were conducted, the $\tau(\dot{\gamma})$ curves did not show any trend to a yield stress.

A power-law model was fitted on the shear rate range $\left[0.1 s^{-1} ; 100 s^{-1}\right]$ which is representative of 

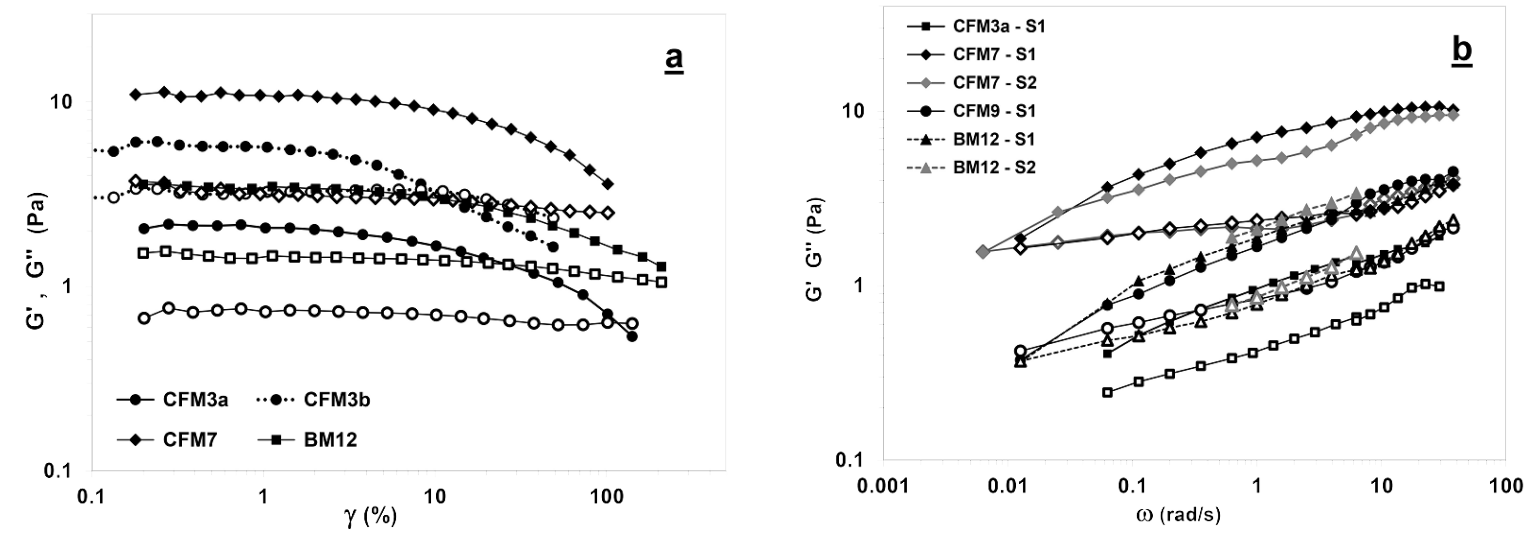

Figure 5.11: Dynamic oscillatory shear tests (filled symbols : elastic modulus $G^{\prime}$, hollow symbols: viscous modulus $G^{\prime \prime}$ ). (a) strain amplitude sweep, (b) frequency sweep.

shear rate involved in the flow. The power-law parameter (index $n$ and consistency $k$ ) are reported in Table 5.3 (from Chatelin and Poncet (2016) for $(n, k)$ values). The power-law indexes $n$ are in the range 0.2 to 0.4 and are thus similar to values obtained by Puchelle et al. (1987), Nielsen et al. (2004), Suk et al. (2009) and Tomaiuolo et al. (2014) while Moores et al. (1992) and Dawson et al. (2003) observed lower $n$-values. Values of consistency $k$, and then for viscosity are also similar. The large shear rate range explored in this study gives an appropriate description of viscosity for the present study, which means this range is coherent with the shear rates observed in the numerical simulations.

Oscillatory measurements are presented in Figure 5.11. Strain sweeps are presented for four mucus (Fig. 5.11-a) and clearly show a large linear domain as it extends until 5 to $10 \%$. Figure 5.11-b presents the storage modulus $G^{\prime}$ and the loss modulus $G^{\prime \prime}$ from a frequency sweep. These curves clearly display the viscoelastic character of mucus: exploring the low frequencies (until $10^{-3} \mathrm{~Hz}$, i.e. more than $1000 \mathrm{~s}$ for a point), the $G^{\prime}(\omega)$ and $G^{\prime \prime}(\omega)$ curves present the typical viscoelastic frequency-evolution: although slightly varying with frequency, these curves cross each other at a frequency $\omega_{c}$ which allows the determination of a characteristic times $T_{c}=1 / \omega_{c}$. This cross frequency was not reached for each mucus, but values or estimation are reported in Table 5.3. They range from $20 s$ up to $100 s$ or more, thus highlighting the precautions to be taken for measurements that must correspond to a steady regime.

As a great number of studies only consider dynamic measurements, it is of interest to examine the validity of steady shear viscosity estimated from these measurements. For polymer solutions and polymer melts, the Cox-Merz rule (Eq. 5.22) is considered as an acceptable way to evaluate viscosity. Parameters $k^{*}$ and $n^{*}$ of the power-laws fitted on $\left|\eta^{*}(\omega)\right|$ data are reported in Table 5.3 and presented in Figure 5.8-a and $\mathrm{b}$ for $k$ vs $k^{*}$ and $n$ vs $n^{*}$ respectively.

Results are slightly different from those extracted from literature: for consistency, which can be regarded as viscosity at $1 s^{-1}, k^{*}$ is similar or higher than $k$. This is coherent with what is known on the validity of the Cox-Merz rule which overestimate the shear viscosity (Picout and Ross-Murphy, 2003). Concerning the power-law index, $n^{*}$ is similar to $n$ for five cases but significantly lower for others, i.e. indicates a more shear-thinning behaviour. Considering both $k^{*}$ and $n^{*}$, this signifies that the difference between dynamic viscosity and shear viscosity becomes increasingly large with decreasing shear rates: considering the viscosity at $1 s^{-1}$, a relative differences of more than $100 \%$ is observed 7 times out of 13 . For a shear rate of $0.01 \mathrm{~s}^{-1}$, this score reaches 9 out of 13 .

Nevertheless, a power-law index of 0.2 (resp. 0.4) leads to changes in viscosity in a ratio 6.3 (resp. 4) over an order of magnitude on shear rate. It follows that variations of viscosity in a flow such that which is considered here are most of the time considerably larger than variations emanating from the discrepancy induced by the Cox-Merz rule. In this sense, dynamic measurements for viscosity can be 


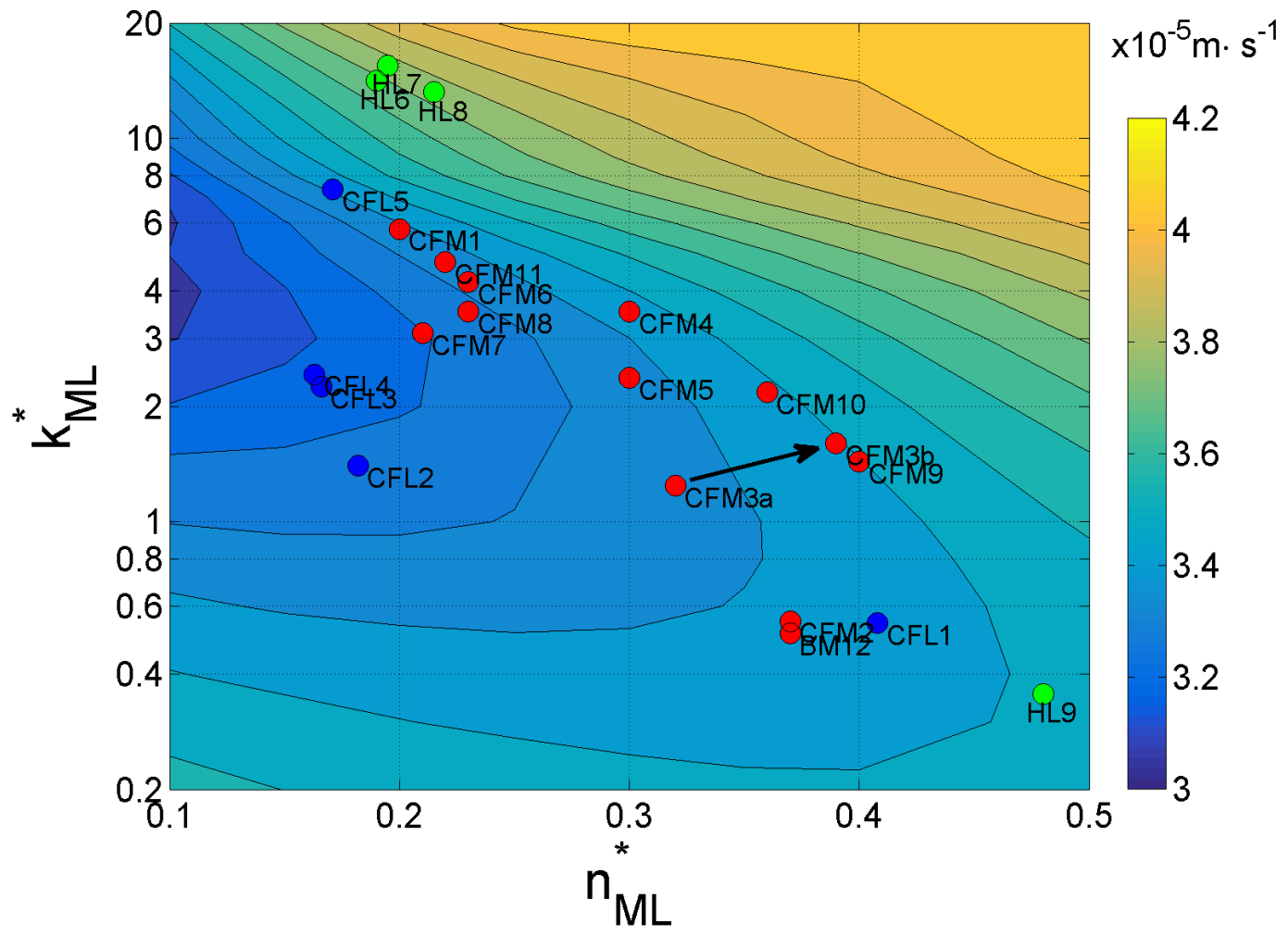

Figure 5.12: Aliases from Tables 5.2 and 5.3. Blue and red markers are Cystic Fibrosis samples respectively from the literature and from our study, while green markers denote healthy samples from the literature.

taken into consideration when shear viscosity cannot be measured.

\subsubsection{Mathematical modelling of the rheology and related numerical analysis}

As presented in the previous section, mucus exhibits a non-Newtonian behaviour with shear-thinning effects. The previous section shows that the power-law is a good representation of the rheological behaviour of the mucus. As presented in Table 5.3 the exponent of the shear rate is negative (since $n$ was identified below unity). From a mathematical and a computational point of view, this can be critical because the viscosity can tend toward infinity when the shear rate tends toward zero, in particular when the flow is at rest (at any given time and given point) or when any infinitely small fluid volume undergoes a solid-like displacement (i.e. with no strain).

In order to avoid the singularity that arises at rest, a limitation of the viscosity at low shear rates is introduced. Furthermore, the aqueous polymer mixture cannot be less viscous than its solvent, which means that at high shear rate, the decreasing viscosity has a lower bound at the PCL viscosity (Newtonian with viscosity equal to that of water at $20^{\circ} \mathrm{C}$ ). Among the different constitutive laws, the Carreau model is interesting as it is a power-law with two Newtonian limits:

$$
\eta(\dot{\gamma})=\eta_{\min }+\left(\eta_{\max }-\eta_{\min }\right)\left[1+(\beta \dot{\gamma})^{2}\right]^{\frac{n^{*}-1}{2}}
$$

The transition between the plateau with viscosity $\eta_{\max }$, and the power-law behaviour occurs at $\dot{\gamma}=$ $1 / \beta$. So $\beta$ can be used for smoothing and avoid the singularity at rest, and therefore can be chosen as large as wanted. The parameter $\eta_{\max }$ is then calculated from the power-law parameters and the $\beta$-value. In our simulations, $\beta$ is set to $4000 \mathrm{~s}^{-1}$, this corresponds to a shear rate transition lower than the lowest 
shear rates explored in mucus rheometry. This $\beta$ value was sufficient to ensure that the results do not depend on the regularisation process. On the other hand, viscosity is limited to values higher than $\eta_{\text {min }}$ (water viscosity here) for high shear rates.

Furthermore, the previous non-Newtonian results are given for the mucus layer and the periciliary fluid layer remains a Newtonian fluid whose viscosity is equal to that of water. Hence it is necessary to model viscosity as a function of $\alpha$ : with a transition from the Newtonian periciliary fluid when $\alpha=0$ to the non-Newtonian Carreau model when $\alpha=1$.

From a numerical point of view, the only difference is the definition of the viscosity which is now a function of the velocity, through the shear rate $\dot{\gamma}(u)=(2 D: D)^{1 / 2}$. The problem to solve is the same as (5.10), except the second equation which is replaced by an $\alpha$-dependant rheological constitutive equation (Chatelin et al., 2016a):

$$
\eta(\dot{\gamma})=\Phi(u, \alpha)=\eta_{P C F}+\left(\eta_{0}(\alpha)-\eta_{P C F}\right)\left[1+(\beta \dot{\gamma})^{2}\right]^{\frac{n^{*}(\alpha)-1}{2}}
$$

where

$$
\eta_{0}(\alpha)=\eta_{P C F}\left(\frac{k_{M L}^{*}}{\eta_{P C F}}\right)^{\alpha} \beta^{\alpha\left(1-n_{M L}^{*}\right)} \text { and } n^{*}(\alpha)=\alpha \cdot n_{M L}^{*}+(1-\alpha)
$$

As mentioned previously, these equations state that the viscosity of the mucus cannot be smaller than the periciliary fluid viscosity, even when the shear rate tends toward infinity. They are introduced in Equation (5.2), which reads:

$$
-\operatorname{div}(2 \eta(\dot{\gamma}) D)+\frac{\mathbf{1}_{B(t)}}{\varepsilon}(u-\bar{u})=f-\nabla p \quad \text { and } \quad \operatorname{div} u=0 \quad \text { in } \Omega
$$

with the adequate boundary and initial conditions, coupled to the model followed by $\alpha$, either the transport Equation (5.12) or the stratified law displayed on Figure 5.3.

Hence the computational algorithm presented in Section \$5.2.2 is slightly modified. Indeed the computation of $\alpha$ remains identical whereas a non-linearity appears in the conservation of momentum equation. This non-linearity is treated "explicitly" in the same fixed point as the projection iterations. Hence the only modification is in the step $2 b$ of this algorithm (see §5.2.2.3) which becomes a multicriteria fixed-point algorithm. Denoting $\eta_{i}=\Phi\left(u_{i}, \alpha\right)$ the new step $2 \mathrm{~b}$ reads:

$$
-\eta_{j} \Delta u_{j+1}^{*}+\frac{\mathbf{1}_{B(t)}}{\varepsilon}\left(u_{j+1}^{*}-\nabla \zeta_{j}-\bar{u}(t)\right)=f+\left(2 D\left(u_{j}\right)+\operatorname{div} u_{k}^{*}\right) \nabla \eta_{j}
$$

The rest of the algorithm presented in Section 5.2.2 remains unchanged. In particular the SMW approach (see §5.2.2.4) is still applicable to compute (5.27) as well as the operator splitting and the Lagrangian computation of the convection of the mucin's ratio.

\subsubsection{Medical outcomes}

Regarding the Newtonian case, a parametric study was performed to investigate the influence of the rheological parameters on the mucociliary clearance efficiency (Chatelin et al., 2016a). The same quantity, defined by Equation (5.21), is computed for this discussion. In Figure 5.12, the results are presented for $169\left(n_{M L}^{*}, k_{M L}^{*}\right)$ pairs using the $\alpha$-dependant rheological constitutive equations previously introduced. The mucus samples presented in the Section 5.3, on Tables 5.2 and 5.3, are also reported on this phase cartography.

This figure clearly shows that the mucociliary transport does not evolves linearly with respect to the power-law coefficients $n^{*}$ and $k^{*}$. This is a direct consequence of the non-linearities of the modelling. In fact, it not meaningful to discuss viscosity variations for shear-thinning fluids and these power-law 


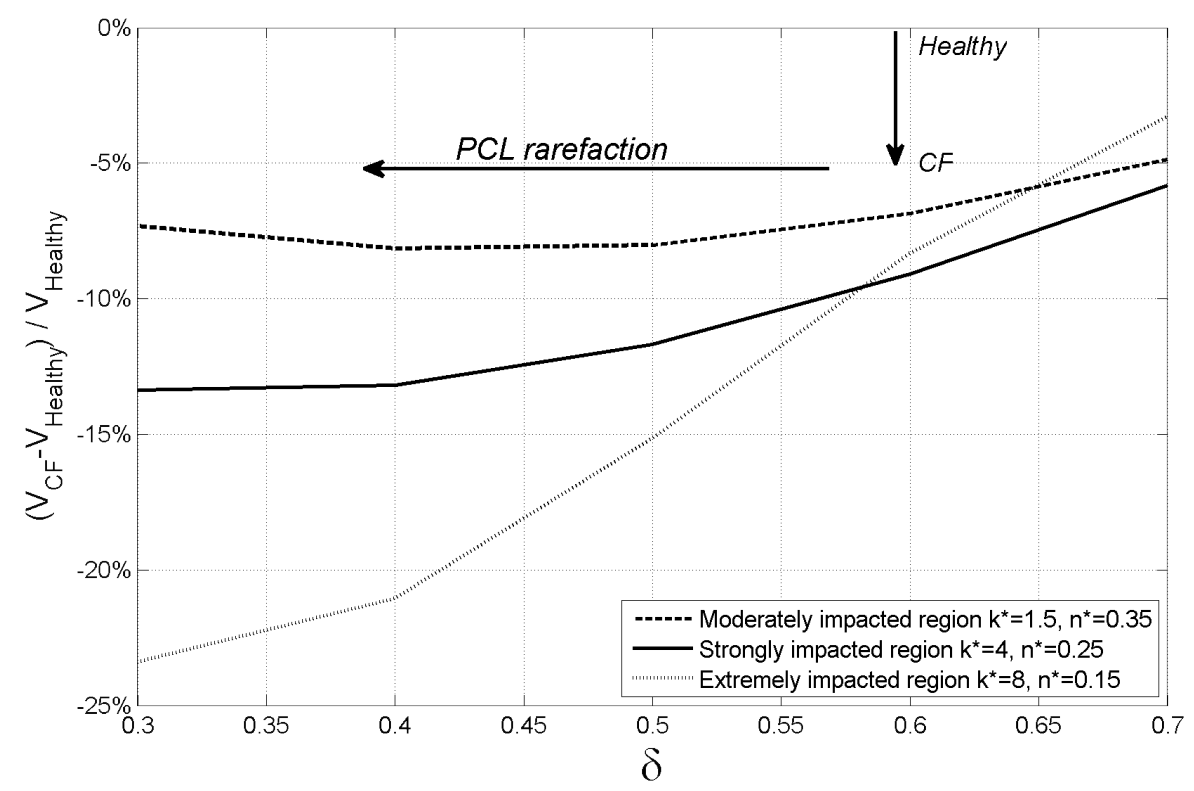

Figure 5.13: Impact of PCL rarefaction ( $\delta$ decreasing from 0.7 et 0.3 ) on mucus mean velocity in percent of healthy mucus velocity. The healthy velocity is the mean velocity of HL6-8 for $\delta=0.6$. The Moderately impacted region corresponds to the samples CFM3,4,5,9,10, the Strongly impacted region corresponds to the samples CFM1,6,7,8,11, and the Extremely impacted region corresponds to the samples CFL5.

coefficients are more significant. It is noteworthy to observe that that the CF mucus generally take lower consistency values $k$ than healthy mucus samples, as well as lower power-law exponent $n$ (except for the HL9).

Figure 5.12 shows that a drop of 6-15\% mobility may be observed for the CF mucus than in the healthy samples. Even if this decrease is not spectacular, it is sufficient to trigger pathological infections such as Pseudomonas aeruginosa or Burkholderia cepacia (Eberl and Tümmler, 2004). Moreover it is important to notice that even if the datum of HL9 (Serisier et al., 2009) is very different from other healthy mucus (Jeanneret-Grosjean et al., 1988; Zayas et al., 1990), the mean mucus velocity remains similar and we can conclude that the associated mucociliary transports are comparable.

Figure 5.12e demonstrates that the shear-thinning behaviour has a very strong effect: when the power-law exponent decreases to 0.2 (which is the measured value for 5 samples), the mucociliary transport is highly deteriorated. This is counterintuitive, but it is the consequence of a higher velocity during the recovery stroke in the mucus layer, since the "apparent viscosity" decreases.

From a clinical point of view, two types of CF mucus can be distinguished: stabilized and decompensated (with acute secondary infections). The mucus of same patient was sampled in both states: two days after hospitalisation (CFM3a, decompensated) and after 17 days of treatment, combining antibiotics and steroids (CFM3b, stabilised). The computations presented in Figure 5.12 clearly show an increase in the mucociliary transport for this patient, thus we conclude that the treatment was beneficial.

Finally, similarly to the Newtonian case, several computations were performed for different heights of periciliary fluid and still 169 values of $\left(n_{M L}^{*}, k_{M L}^{*}\right)$ pairs - see Chatelin et al. (2016a) for details. It was demonstrated that this pericilary fluid height has a very important effect on the mucociliary transport with up to a $21 \%$ decrease in mucus mean velocity. This parameter represents the effect of a variable CFTR deficiency (Thornton and Sheehan, 2004; Ehre et al., 2014) and it also shows the lack of mucociliary clearance in cystic fibrosis, allowing pathological proliferations. 
This effect had been summarized in Figure 5.13, where three groups are displayed:

- A group $k^{*}=1.5, n^{*}=0.35$, corresponding to a centre of gravity of samples CFM3-5,9,10, which is moderately impacted by PCL rarefaction,

- A group $k^{*}=4, n^{*}=0.25$, corresponding to a centre of gravity of samples CFM1,6-8,11, which is strongly impacted by PCL rarefaction, decreased up to $-13 \%$ compared to healthy cases, which means a $-6 \%$ due to lack of mucociliary clearance efficiency, made worse by $-7 \%$ due to PCL height decrease.

- A location $k^{*}=8, n^{*}=0.15$, corresponding to case CFL5 reported in Dawson et al. (2003), corresponding to a situation extremely impacted by PCL rarefaction, with a decrease of $-23 \%$ compared the healthy configuration.

\subsection{Concluding remarks}

The lung is resistant to damages following exposure to pathogens and more or less toxic chemical pollutants in inhaled air. Airway mucus represents the first line of defense. Inhaled particles (allergens, dust, micro-organisms, and cellular debris) trapped in the mucus gel are removed from the respiratory tract by the mucociliary clearance. Mucus cleans airways, as it flows from either the tracheobronchial tree or upper airways toward the pharynx, where it is swallowed (or expectorated).

Ciliary motions enable mucus propulsion in the human respiratory tract. The beating cycle of respiratory cilia is composed of 3 phases: a rest phase, a recovery stroke, and an effective stroke. During the recovery stroke, cilia (height $1-7 \mu \mathrm{m}$ according to the location in the respiratory tract and investigators) sweep backward remaining close to the epithelium and immersed in the periciliary fluid without contact with mucus. Cilia of a patch of ciliated cells form a coordinated beating cluster (beating frequency 10-20 $\mathrm{Hz}$ ). The cilium tips impact mucus during a short phase of its beating cycle. Cilia are more rigid in the forward power stroke and more flexible in recovery stroke in preparation for the next effective stroke.

The present mathematical model of airway surface liquid dynamics relies on a heterogeneous fluid that moves due to beating deformable cilia. The airway surface liquid nature evolves continuously in both composition and rheology from the periciliary fluid to the mucus. Within the near-wall fluid surrounding the deformable cilia, the mucin polymers released by the epithelium further hydrate. Once they reached a certain distance normal to the wetted surface of the respiratory epithelium, which is slightly lower than the cilium height, mucin polymers accumulate, engendering a mucin-concentrated phase. In this region, mucin polymers interweave and eventually cross-link. This evolving nature of the airway surface liquid affects its rheology. For example, for a airway surface liquid thickness of $12 \mu \mathrm{m}$ and cilium height of 8 $\mu \mathrm{m}$, the thickness of the periciliary fluid and mucus are equal to 7 and $5 \mu \mathrm{m}$, respectively.

The rheological behaviour is associated with two parameters of interest, the fluid consistency index $k$ and the power law index $n$ that describes the difference between the two asymptotic behaviours ( $\mu_{0}$ and $\mu_{\infty}$ ) and the slope of the shear rate-viscosity curve between these two asymptotic behaviours.

The numerical model is combined with an experimental study of the rheology of sputum from cystic fibrosis patients. The proposed robust numerical method handle variations in shear rate and viscosity of more than 5 orders of magnitude. It leads to a cartography that allows to discuss major issues on defective mucociliary clearance observed in cystic fibrosis during periodic check-up of the disease in a steady clinical state (i.e., in the absence of infection exacerbation) for prognosis. 


\section{Bibliography}

Adams, J. C. (1989). mudpack: Multigrid portable fortran software for the efficient solution of linear elliptic partial differential equations. Applied Mathematics and Computation, 34(2, Part 2):113-146.

Adams, R. A. and Fournier, J. J. F. (2003). Sobolev Spaces. Academic Press.

Aitken, M. L., Bellon, G., De Boeck, K., Flume, P. A., Fox, H. G., Geller, D. E., Haarman, E. G., Hebestreit, H. U., Lapey, A., Schou, I. M., Zuckerman, J. B., and Charlton, B. (2012). Long-Term Inhaled Dry Powder Mannitol in Cystic Fibrosis. American Journal of Respiratory and Critical Care Medicine, 185(6):645-652.

Angot, P., Bruneau, C.-H., and Fabrie, P. (1999). A penalization method to take into account obstacles in incompressible viscous flows. Numerische Mathematik, 81(4):497-520.

App, E. M., Kieselmann, R., Reinhardt, D., Lindemann, H., Dasgupta, B., King, M., and Brand, P. (1998). Sputum rheology changes in cystic fibrosis lung disease following two different types of physiotherapy : Flutter vs autogenic drainage. Chest, 114(1):171-177.

Barnes, H. A., Hutton, J. F., and Walters, K. (1989). An introduction to rheology. Elsevier.

Beale, J. T. and Majda, A. (1981). Rates of convergence for viscous splitting of the navier-stokes equations. Mathematics of Computation, 37(156):243-259.

Besseris, G. J. and Yeates, D. B. (2007). Rotating magnetic particle microrheometry in biopolymer fluid dynamics: Mucus microrheology. The Journal of Chemical Physics, 127(10):105106-105106-17.

Bilton, D., Robinson, P., Cooper, P., Gallagher, C. G., Kolbe, J., Fox, H., Jaques, A., and Charlton, B. (2011). Inhaled dry powder mannitol in cystic fibrosis: an efficacy and safety study. European Respiratory Journal, 38(5):1071-1080.

Bird, R., Armstrong, R., and Hassager, O. (1977). Dynamics of Polymeric Liquids. John Wiley Sons.

Blake, J. (1972). A model for the micro-structure in ciliated organisms. Journal of Fluid Mechanics, 55(01):1-23.

Boyer, F. and Fabrie, P. (2005). Eléments d'analyse pour l'étude de quelques modèles d'écoulements de fluides visqueux incompressibles. Springer.

Broughton-Head, V. J., Shur, J., Carroll, M. P., Smith, J. R., and Shute, J. K. (2007). Unfractionated heparin reduces the elasticity of sputum from patients with cystic fibrosis. AJP: Lung Cellular and Molecular Physiology, 293(5):L1240-L1249.

Button, B., Cai, L.-H., Ehre, C., Kesimer, M., Hill, D. B., Sheehan, J. K., Boucher, R. C., and Rubinstein, M. (2012). Periciliary brush promotes the lung health by separating the mucus layer from airway epithelia. Science (New York, N.Y.), 337(6097):937-941.

Carbou, G. and Fabrie, P. (2003). Boundary layer for a penalization method for viscous incompressible flow. Differential Equations, 8(12):1453-1480. 
Chatelin, R. (2013). Méthodes numériques pour l'écoulement de Stokes 3D: fluides à viscosité variable en géométrie complexe mobile; application aux fluides biologiques. $\mathrm{PhD}$ thesis, Université Toulouse 3 Paul Sabatier.

Chatelin, R., Anne-Archard, D., Murris-Espin, M., Thiriet, M., and Poncet, P. (2016a). Numerical and experimental investigation of mucociliary clearance breakdown in cystic fibrosis. Submitted.

Chatelin, R. and Poncet, P. (2013). A hybrid grid-particle method for moving bodies in 3D stokes flow with variable viscosity. SIAM Journal on Scientific Computing, 35(4):B925-B949.

Chatelin, R. and Poncet, P. (2014). Hybrid grid-particle methods and penalization: A ShermanMorrison-Woodbury approach to compute 3D viscous flows using FFT. Journal of Computational Physics, 269:314-328.

Chatelin, R. and Poncet, P. (2016). A parametric study of mucociliary transport by numerical simulations of 3d non-homogeneous mucus. Journal of Biomechanics, 49(9):1772-1780.

Chatelin, R., Poncet, P., Didier, A., Murris-Espin, M., Anne-Archard, D., and Thiriet, M. (2015). Mucus and ciliated cells of human lung: Splitting strategies for particle methods and $3 \mathrm{~d}$ stokes flows. Procedia IUTAM, 18:114-122.

Chatelin, R., Sanchez, D., and Poncet, P. (2016b). Analysis of the penalized 3d variable viscosity stokes equations coupled to diffusion and transport. ESAIM: Mathematical Modelling and Numerical Analysis, 50(2):565-591.

Clancy, J. P. and Jain, M. (2012). Personalized Medicine in Cystic Fibrosis. American Journal of Respiratory and Critical Care Medicine, 186(7):593-597.

Cone, R. (2005). Mucosal Immunology, chapter 4: Mucus, pages 49-72. Elsevier Academic Press.

Cortez, R., Fauci, L., and Medovikov, A. (2005). The method of regularized stokeslets in three dimensions: Analysis, validation, and application to helical swimming. Physics of Fluids, 17(3):031504.

Cottet, G.-H. and Koumoutsakos, P. D. (2000). Vortex Methods: Theory and Practice. Cambridge University Press.

Craster, R. V. and Matar, O. K. (2000). Surfactant transport on mucus films. Journal of Fluid Mechanics, 425:235-258.

Dautray, R. and Lions, J. L. (2000). Mathematical Analysis and Numerical Methods for Science and Technology: Volume 1-6. Springer.

Daviskas, E., Anderson, S. D., and Young, I. H. (2010). Effect of mannitol and repetitive coughing on the sputum properties in bronchiectasis. Respiratory Medicine, 104(3):371-377.

Dawson, M., Wirtz, D., and Hanes, J. (2003). Enhanced Viscoelasticity of Human Cystic Fibrotic Sputum Correlates with Increasing Microheterogeneity in Particle Transport. Journal of Biological Chemistry, 278(50):50393-50401.

Deneuville, E., Perrot-Minot, C., Pennaforte, F., Roussey, M., Zahm, J.-M., Clavel, C., Puchelle, E., and Bentzmann, S. D. (1997). Revisited physicochemical and transport properties of respiratory mucus in genotyped cystic fibrosis patients. American Journal of Respiratory and Critical Care Medicine, 156:166-172.

Doring, G., Flume, P., Heijerman, H., and Elborn, J. S. (2012). Treatment of lung infection in patients with cystic fibrosis: Current and future strategies. Journal of Cystic Fibrosis, 11(6):461-479. 
Eberl, L. and Tümmler, B. (2004). Pseudomonas aeruginosa and burkholderia cepacia in cystic fibrosis: genome evolution, interactions and adaptation. International Journal of Medical Microbiology, 294(2-3):123-131.

Edwards, P. A. and Yeates, D. B. (1992). Magnetic rheometry of bronchial mucus. In Viscoelasticity of Biomaterials, volume 489 of ACS Symposium Series, pages 249-267. American Chemical Society.

Ehre, C., Ridley, C., and Thornton, D. J. (2014). Cystic fibrosis: An inherited disease affecting mucinproducing organs. The International Journal of Biochemistry \& Cell Biology, 52:136-145.

Enault, S., Lombardi, D., Poncet, P., and Thiriet, M. (2010). Mucus dynamics subject to air and wall motion. ESAIM Proceedings, 30:124-141.

Fahy, J. V. and Dickey, B. F. (2010). Airway mucus function and dysfunction. The New England journal of medicine, 363(23):2233-2247. PMID: 21121836.

Farrell, P. M. (2008). The prevalence of cystic fibrosis in the european union. Journal of Cystic Fibrosis, 7:450-453.

Fauci, L. J. and Dillon, R. (2006). Biofluidmechanics of reproduction. Annual Review of Fluid Mechanics, 38(1):371-394.

Fulford, G. and Blake, J. (1986). Muco-ciliary transport in the lung. Journal of Theoretical Biology, 121(4):381-402.

Gastaldi, A. C., Jardim, J. R., and King, M. (2000). The influence of temperature and length of time of storage of frog mucus samples. Biorheology, 37:203-211.

Gheber, L. and Priel, Z. (1997). Extraction of cilium beat parameters by the combined application of photoelectric measurements and computer simulation. Biophysical Journal, 72(1):449-462.

Guermond, J., Minev, P., and Shen, J. (2006). An overview of projection methods for incompressible flows. Computer Methods in Applied Mechanics and Engineering, 195:6011-6045.

Hager, W. W. (1989). Updating the inverse of a matrix. SIAM Rev., 31(2):221-239.

Haq, I. J., Gray, M. A., Garnett, J. P., Ward, C., and Brodlie, M. (2015). Airway surface liquid homeostasis in cystic fibrosis: pathophysiology and therapeutic targets. Thorax, pages thoraxjnl-2015-207588.

Hill, D. B., Swaminathan, V., Estes, A., Cribb, J., O'Brien, E. T., Davis, C. W., and Superfine, R. (2010). Force Generation and Dynamics of Individual Cilia under External Loading. Biophysical Journal, 98(1):57-66.

Hoffman, L. R. and Ramsey, B. W. (2013). Cystic fibrosis therapeutics: The road ahead. Chest, 143(1):207-213.

Hussong, J., Breugem, W.-P., and Westerweel, J. (2011). A continuum model for flow induced by metachronal coordination between beating cilia. Journal of Fluid Mechanics, 684:137-162.

Innes, A. L., Carrington, S. D., Thornton, D. J., Kirkham, S., Rousseau, K., Dougherty, R. H., Raymond, W. W., Caughey, G. H., Muller, S. J., and Fahy, J. V. (2009). Ex Vivo Sputum Analysis Reveals Impairment of Protease-dependent Mucus Degradation by Plasma Proteins in Acute Asthma. American Journal of Respiratory and Critical Care Medicine, 180(3):203-210.

Jayathilake, P., Le, D., Tan, Z., Lee, H., and Khoo, B. (2015). A numerical study of muco-ciliary transport under the condition of diseased cilia. Computer Methods in Biomechanics and Biomedical Engineering, 18(9):944-951. 
Jayathilake, P., Tan, Z., Le, D., Lee, H., and Khoo, B. (2012). Three-dimensional numerical simulations of human pulmonary cilia in the periciliary liquid layer by the immersed boundary method. Computers \& Fluids, 67(0):130-137.

Jeanneret-Grosjean, A., King, M., Michoud, M. C., Liote, H., and Amyot, R. (1988). Sampling Technique and Rheology of Human Tracheobronchial Mucus. American Review of Respiratory Disease, 137(3):707-710.

Jeffries, J. L., Jia, J., Choi, W., Choe, S., Miao, J., Xu, Y., Powell, R., Lin, J., Kuang, Z., Gaskins, H. R., and Lau, G. W. (2015). Pseudomonas aeruginosa pyocyanin modulates mucin glycosylation with sialyl-Lewisx to increase binding to airway epithelial cells. Mucosal Immunology.

King, M. (1980). Rheological requirements for poptimal clearance of secretions: ciliary transport cversus cough. European Journal of Respiratory Diseases, 110:39-45.

King, M. (1981). Is cystic fibrosis mucus abnormal? Pediatric Research, 15:120-122.

King, M. and Macklem, P. T. (1977). Rheological properties of microliter quantities of normal mucus. Journal of Applied Phycology, 42(6):797-802.

Kurbatova, E. V., Cegielski, J. P., Lienhardt, C., Akksilp, R., Bayona, J., Becerra, M. C., Caoili, J., Contreras, C., Dalton, T., Danilovits, M., Demikhova, O. V., Ershova, J., Gammino, V. M., Gelmanova, I., Heilig, C. M., Jou, R., Kazennyy, B., Keshavjee, S., Kim, H. J., Kliiman, K., Kvasnovsky, C., Leimane, V., Mitnick, C. D., Quelapio, I., Riekstina, V., Smith, S. E., Tupasi, T., van der Walt, M., Vasilyeva, I. A., Via, L. E., Viiklepp, P., Volchenkov, G., Walker, A. T., Wolfgang, M., Yagui, M., and Zignol, M. (2015a). Sputum culture conversion as a prognostic marker for end-of-treatment outcome in patients with multidrug-resistant tuberculosis: a secondary analysis of data from two observational cohort studies. The Lancet Respiratory Medicine, 3(3):201-209.

Kurbatova, P., Bessonov, N., Volpert, V., Tiddens, H., Cornu, C., Nony, P., and Caudri, D. (2015b). Model of mucociliary clearance in cystic fibrosis lungs. Journal of Theoretical Biology, 372:81 - 88.

Lai, S. K., Wang, Y.-Y., Wirtz, D., and Hanes, J. (2009). Micro- and macrorheology of mucus. Advanced Drug Delivery Reviews, 61(2):86-100.

Lee, W., Jayathilake, P., Tan, Z., Le, D., Lee, H., and Khoo, B. (2011). Muco-ciliary transport: Effect of mucus viscosity, cilia beat frequency and cilia density. Computers \& Fluids, 49(1):214-221.

Li, Z., Favier, J., D'Ortona, U., and Poncet, S. (2016). An immersed boundary-lattice Boltzmann method for single- and multi-component fluid flows. Journal of Computational Physics, 304:424-440.

Liron, N. and Rozenson, M. (1983). Muco-ciliary transport. J. submicrosc. Cytol, 15(1):317-321.

Lopez-Vidriero, M. T. and Reid, L. (1978). Chemical markers of mucous and serum glycoproteins and their relation to viscosity in mucoid and purulent sputum from various hypersecretory diseases. American Review of Respiratory Disease, 117:465-477.

Lukens, S., Yang, X., and Fauci, L. (2010). Using Lagrangian coherent structures to analyze fluid mixing by cilia. Chaos: An Interdisciplinary Journal of Nonlinear Science, 20(1):017511.

Mall, M. A. and Hartl, D. (2014). CFTR: cystic fibrosis and beyond. European Respiratory Journal, 44(4):1042-1054.

McShane, P. J., Naureckas, E. T., Tino, G., and Strek, M. E. (2013). Non-Cystic Fibrosis Bronchiectasis. American Journal of Respiratory and Critical Care Medicine, 188(6):647-656.

Mitran, S. M. (2007). Metachronal wave formation in a model of pulmonary cilia. Computers \& Structures, 85:763-774. 
Monaghan, J. (1985). Extrapolating b splines for interpolation. Journal of Computational Physics, 60(2):253-262.

Montenegro-Johnson, T. D., Smith, D. J., and Loghin, D. (2013). Physics of rheologically enhanced propulsion: Different strokes in generalized stokes. Physics of Fluids, 25(8):081903.

Moores, C., Hudson, N. E., and Davies, A. (1992). The effect of high-frequency ventilation on nonNewtonian properties of bronchial mucus. Respiratory Medicine, 86(2):125-130.

Nielsen, H., Hvidt, S., Sheils, C. A., and Janmey, P. A. (2004). Elastic contributions dominate the viscoelastic properties of sputum from cystic fibrosis patients. Biophysical Chemistry, 112(2-3):193200.

Peskin, C. S. (2003). The immersed boundary method. Acta Numerica, 11.

Picout, D. R. and Ross-Murphy, S. B. (2003). Rheology of Biopolymer Solutions and Gels. The Scientific World Journal, 3:105-121.

Puchelle, E., Zahm, J. M., Duvivier, C., Didelon, J., Jacquot, J., and Quemada, D. (1985). Elastothixotropic properties of bronchial mucus and polymer analogs. i- experimental results. Biorheology, 22:415-423.

Puchelle, E., Zahm, J. M., and Quemada, D. (1987). Rheological properties controlling mucociliary frequency and respiratory mucus transport. Biorheology, 24(6):557-563. PMID: 3502756.

Quon, B. S. and Rowe, S. M. (2016). New and emerging targeted therapies for cystic fibrosis. BMJ, 352:i859.

Redding, G. J. (2008). Physical and transport properties of sputum from children with idiopathic bronchiectasis. CHEST Journal, 134(6):1129.

Rubin, B. K., Ramirez, O., Zayas, J. G., Finegan, B., and King, M. (1990). Collection and Analysis of Respiratory Mucus from Subjects without Lung Disease. American Review of Respiratory Disease, 141(4_pt_1):1040-1043.

Sanders, N. N., Smedt, S. C. D., Rompaey, E. V., Simoens, P., Baets, F. D., and Demeester, J. (2000). Cystic fibrosis sputum. a barrier to the transport of nanospheres. American Journal of Respiratory and Critical Care Medicine, 162:1905-1911.

Sanderson, M. J. and Sleigh, M. A. (1981). Ciliary activity of cultured rabbit tracheal epithelium: beat pattern and metachrony. Journal of Cell Science, 47(1):331-347.

Schuster, B. S., Suk, J. S., Woodworth, G. F., and Hanes, J. (2013). Nanoparticle diffusion in respiratory mucus from humans without lung disease. Biomaterials, 34(13):3439-3446.

Sedaghat, M. H., Shahmardan, M. M., Norouzi, M., Nazari, M., and Jayathilake, P. G. (2016). On the effect of mucus rheology on the muco-ciliary transport. Mathematical Biosciences, 272:44-53.

Serisier, D. J., Carroll, M. P., Shute, J. K., and Young, S. A. (2009). Macrorheology of cystic fibrosis, chronic obstructive pulmonary disease \&amp; normal sputum. Respiratory Research, 10(1):63.

Shah, P. L., Scott, S. F., Knight, R. A., Marriott, C., Ranasinha, C., and Hodson, M. E. (1996). In vivo effects of recombinant human DNase i on sputum in patients with cystic fibrosis. Thorax, 51(2):119125.

Silberberg, A. (1983). Biorheological matching : mucociliary interaction and epithelial clearance. Biorheology, 20:215-222. 
Simons, J., Fauci, L., and Cortez, R. (2015). A fully three-dimensional model of the interaction of driven elastic filaments in a Stokes flow with applications to sperm motility. Journal of Biomechanics, 48(9):1639-1651.

Smith, D., Gaffney, E., and Blake, J. (2007a). Discrete cilia modelling with singularity distributions: Application to the embryonic node and the airway surface liquid. Bulletin of Mathematical Biology, 69(5):1477-1510.

Smith, D., Gaffney, E., and Blake, J. (2007b). A viscoelastic traction layer model of muco-ciliary transport. Bulletin of Mathematical Biology, 69(1):289-327.

Smith, D., Gaffney, E., and Blake, J. (2008). Modelling mucociliary clearance. Respiratory Physiology \& Neurobiology, 163:178-188.

Suk, J. S., Lai, S. K., Wang, Y.-Y., Ensign, L. M., Zeitlin, P. L., Boyle, M. P., and Hanes, J. (2009). The penetration of fresh undiluted sputum expectorated by cystic fibrosis patients by non-adhesive polymer nanoparticles. Biomaterials, 30(13):2591-2597.

Swarztrauber, P. and Sweet, R. (1975). Efficient FORTRAN subprograms for the solution of elliptic partial differential equations (abstract). SIGNUM Newsl., 10(4).

Tabatabaei, S., Jahromi, H., Webster, M., Williams, P., Holder, A., Lewis, K., Davies, G., Griffin, L., Ebden, P., and Askill, C. (2015). A CaBER computational-experimental rheological study on human sputum. Journal of Non-Newtonian Fluid Mechanics, 222:272-287.

Thiriet, M. (2012). Tissue Functioning and Remodeling in the Circulatory and Ventilatory Systems. Number v.5 in Biomathematical and Biomechanical Modeling of the Circulatory and Ventilatory Systems. Springer, Dordrecht.

Thomas, R. H. and Walters, K. (1966). The unsteady motion of a sphere in an elastico-viscous liquid. Rheologica Acta, 5(1):23-27.

Thornton, D. J. and Sheehan, J. K. (2004). From mucins to mucus toward a more coherent understanding of this essential barrier. Proceedings of the American Thoracic Society, 1(1):54-61.

Tomaiuolo, G., Rusciano, G., Caserta, S., Carciati, A., Carnovale, V., Abete, P., Sasso, A., and Guido, S. (2014). A New Method to Improve the Clinical Evaluation of Cystic Fibrosis Patients by Mucus Viscoelastic Properties. PLOS ONE, 9(1):e82297.

Twigg, M. S., Brockbank, S., Lowry, P., FitzGerald, S. P., Taggart, C., Weldon, S., ad, Twigg, M. S., Brockbank, S., Lowry, P., FitzGerald, S. P., Taggart, C., Weldon, S., and ad (2015). The Role of Serine Proteases and Antiproteases in the Cystic Fibrosis Lung, The Role of Serine Proteases and Antiproteases in the Cystic Fibrosis Lung. Mediators of Inflammation, Mediators of Inflammation, 2015, 2015:e293053.

Yankaskas, J. R., Marshall, B. C., Sufian, B., Simon, R. H., and Rodman, D. (2004). Cystic fibrosis adult care: Consensus conference report. Chest, 125(1_suppl):1S-39S.

Yeates, D. B., Besseris, G. J., and Wong, L. B. (1997). The Lung: Scientific Foundations, chapter 33 : Physicochemical properties of mucus and its propulsion, pages 487-503. Lippincot-Raven Publishers, Philadelphia.

Zayas, J. G., Man, G. C. W., and King, M. (1990). Tracheal Mucus Rheology in Patients Undergoing Diagnostic Bronchoscopy: Interrelations with Smoking and Cancer. American Review of Respiratory Disease, 141(5_pt_1):1107-1113.

Zayas, J. G., Rubin, B. K., York, E. L., Lien, D. C., and King, M. (1999). Bronchial mucus properties in lung cancer: Relationship with site of lesion. Canadian Respiratory Journal, 6(3):246-252. 
Zemanick, E. T., Harris, J. K., Conway, S., Konstan, M. W., Marshall, B., Quittner, A. L., Retsch-Bogart, G., Saiman, L., and Accurso, F. J. (2010). Measuring and improving respiratory outcomes in cystic fibrosis lung disease: Opportunities and challenges to therapy. Journal of Cystic Fibrosis, 9(1):1-16.

Zielenski, J. (2000). Genotype and Phenotype in Cystic Fibrosis. Respiration, 67(2):117-133.

Ziemann, F., Radler, J., and Sackmann, E. (1994). Local measurements of viscoelastic moduli of entangled actin networks using an oscillating magnetic bead micro-rheometer. Biophysical Journal, 66(6):2210-2216. 